\title{
Modeling Liver Biology and the Tissue Response to Injury in Bioprinted Human Liver Tissues
}

\author{
Kelsey Retting, ${ }^{1}$ Dwayne Carter, ${ }^{1, \star}$ Candace Crogan-Grundy,, ${ }^{1, *}$ Chirag Khatiwala, ${ }^{1, *}$ \\ Leah Norona, ${ }^{2, *}$ Elizabeth Paffenroth, ${ }^{1, *}$ Umesh Hanumegowda,, ${ }^{3, *}$ Alice Chen, \\ Lisa Hazelwood, Lois Lehman-McKeeman, ${ }^{3}$ and Sharon Presnell ${ }^{1}$
}

\begin{abstract}
The rapid advancement of additive manufacturing technologies and their extension into biological systems have led to the emergence of a new category of cell-based models, namely bioprinted tissues. By combining automation and spatially controlled deposition of distinct components, such as cells, hydrogels, and mixtures thereof, the tissue engineer can now design and build tissues with far more control over the architecture of the end product. Bioprinted human liver tissues (BHLT) were one of the first commercial examples of a tissue model fabricated from three or more specific cell types using a custom-built robotic bioprinting platform. Significant progress has been made toward characterizing BHLT and evaluating their performance in a broad spectrum of applications, including in vitro safety and efficacy testing, and disease modeling. Like many multicellular and/or three-dimensional liver tissue models, BHLT exhibit enhanced longevity and persistent liver functions (protein synthesis, metabolism) compared with traditional monocellular or two-dimensional systems. BHLT are a compelling option for many in vitro applications due to several factors: (1) they contain cell-cell interfaces with direct contact between multiple cell types without interference from culture surfaces (polystyrene or polymeric membranes); (2) they have sufficient biomass to enable isolation of nucleic acids or proteins for use in genomic or proteomic analyses; and (3) they are compatible with histology techniques and thus enable detection of outcomes that require histological endpoints for interpretation, such as liver fibrosis and nonalcoholic steatohepatitis. The advantages and limitations of BHLT are highlighted herein through a series of exemplary case studies and related discussion.
\end{abstract}

Keywords: bioprinted, fibrosis, human, liver, model, NAFLD

\section{Introduction}

T HE DESIRE TO CREATE more complex three-dimensional (3D) structures with user-controlled placement of multiple cellular and/or hydrogel components led to the development of early prototype bioprinters that were used to create cell-comprising structures such as tissue sheets and simple tubes in the laboratory. Two seminal publications described the construction of spatially controlled 3D structures from cellular and/or biomaterial building blocks, using distinct methods. ${ }^{1,2}$ Forgacs and colleagues demonstrated that individual aggregates of mammalian cells could be formed and dispensed in a controlled manner and that they would ultimately merge with each other to create a higher order tissue structure based on physical properties akin to the welldescribed interactions of adjacent liquid droplets. ${ }^{1,3,4}$ Smith et al. used a laboratory-built system to dispense mammalian cells suspended in hydrogel components onto a variety of material substrates to create patterned cell-containing structures that persisted in vitro for weeks. ${ }^{2}$

The clear potential of these approaches sparked robust development and commercialization efforts so that a growing number of bioprinters and related consumables, such as preformulated bioinks comprised of cells, hydrogels, or cell/

\footnotetext{
${ }^{1}$ Organovo, Inc., San Diego, California.

${ }^{2}$ Curriculum in Toxicology, Eshelman School of Pharmacy, University of North Carolina at Chapel Hill, Chapel Hill, North Carolina.

${ }^{3}$ Pharmaceutical Candidate Optimization, Bristol-Myers Squibb, Philadelphia, Pennsylvania.

${ }^{4}$ Liver Disease and Fibrosis Discovery, AbbVie, Inc., Chicago, Illinois.

*These authors contributed equally to this work.
}

(c) Kelsey Retting et al., 2018; Published by Mary Ann Liebert, Inc. This Open Access article is distributed under the terms of the Creative Commons License (http://creativecommons.org/licenses/by/4.0), which permits unrestricted use, distribution, and reproduction in any medium, provided the original work is properly cited. 
hydrogel mixtures (approaches and compositions reviewed in Refs. ${ }^{5-7}$ ), are available on the market for researchers and manufacturers.

Once bioprinting systems were available, discovery and development efforts expanded rapidly to focus on the outputs from bioprinting, namely bioprinted tissues (reviewed in Nguyen and Pentoney ${ }^{8}$ ). The design of any new tissue requires significant effort with respect to cell sourcing, defining requirements and limitations for fabrication and tissue design parameters, and the development of companion products and strategies for the maintenance of the tissue post-fabrication including media formulations, feeding strategies, and the identification of suitable culture vessel for maintaining the tissue. Many specialized cell types, such as hepatocytes, have limited tolerances for shear forces and physical compression as well as extremely high metabolic requirements for the maintenance of function 9 ; thus, extensive experimentation is generally required to arrive at a tissue design that is executable and results in retention of target functions.

One critical limitation of bioprinting at the current time is the need to balance resolution or feature size and print speed; designs with high-resolution intricate features will require longer print times to create larger scale tissues. The finest resolution achievable will depend on multiple factors, including tolerances of the specific cell types, the instrument platform, and hydrogel components, but ranges from 10 to $500 \mu \mathrm{m}$, depending on the mode of deposition (i.e., ink-jet, extrusion, acoustic transfer, or laser-driven transfer). ${ }^{8,10}$ Thus, it is important to understand that the finished bioprinted tissue product, regardless of how it was bioprinted, is formed through a combination of directed assembly and self-assembly, with fine features such as microvascular structures forming secondary to the initial bioprinting steps. Moreover, once tissues are formed, significant work is required to develop and validate means of interrogating these "nonstandard" 3D tissue systems with histological, biochemical, and molecular assays.

Bioprinted human liver tissues (BHLT) were among the first bioprinted tissues to be applied in a commercial setting for applications in basic biology, toxicology, and disease modeling (Norona et al., "Bioprinted human liver provides insight into the role of Kupffer cells in modulating early events in TGFB1 and methotrexate-induced fibrotic injury," submitted). ${ }^{8,11-15}$ For this application-focused review, we have concentrated primarily on a commercially available BHLT, for which multiple case studies and outcomes have been reported. However, it should be recognized that this is a rapidly evolving field with multiple academic and industrial laboratories pursuing the fabrication of liver tissues by a variety of bioprinting approaches, although published reports are limited. ${ }^{16-21}$

BHLT have been constructed from primary liver cells, cell lines, and iPSC-derived cells, although the majority of reported efforts have employed primary cells due to the unique and specialized metabolic requirements of hepatocytes that are difficult to achieve with cell lines, such as HepG2 or induced pluripotent stem cells (iPSC)-derived hepatocyte-like cells. Once tissue design and fabrication method have been established, interchange of cells (i.e., primary vs. iPSC, or use of primary cells from a different donor) is achievable, although some optimization may be required depending on the application.

The BHLT discussed herein are composed of primary human hepatocytes (HC), primary hepatic stellate cells
(HSC), and primary endothelial cells (EC). Kupffer cells (KC) are not required for tissue formation or function but may be added to enhance the tissue capacity for immunemediated functions (i.e., cytokine production, matrix metalloproteinase production, phagocytosis). As shown in Figure 1, BHLT are bioprinted directly into a standard culture vessel with access to media on all sides. The distinct bioprinted elements coalesce during the first 24-60 hours post-fabrication to form a $3 \mathrm{D}$ tissue structure that is compartmentalized and almost fully cellular. Only the bottom-most layer of cells within the tissue come into direct contact with polymeric tissue culture surfaces and the physical and biochemical forces exerted by those materials. The resulting proximity of cells within the structure fosters the development of tissue-like features, including tight junctions, bile canaliculi, and microvascular structures. It should be noted, however, that current generation BHLT are not replicas of native human liver tissue; while they possess many relevant cell-cell interactions and tissue features, they are typically lacking true lobular structure with sinusoidal blood flow and other cell types, such as cholangiocytes. The compartmentalized nature of the tissues (i.e., alternating zones enriched for either $\mathrm{HC}$ or $\mathrm{HSC}+\mathrm{EC}$ ) is retained post-fabrication and is confirmed with $\mathrm{HC}$-specific immunolabeling. ${ }^{8,13}$ As with many multicellular and/or 3D in vitro models comprising hepatocytes, the metabolic and synthetic functions of BHLT are maintained for a greater length of time $(\sim 2-4 \times)$ than is routinely reported for standard hepatocyte monocultures or collagen gel sandwich cultures, ${ }^{13}$ including CYP450 expression and activity (Fig. 1C, D).

In addition to the functional longevity, BHLT offer some additional advantages for in vitro applications. The inclusion of two or more nonparenchymal cell (NPC) types, that is, $\mathrm{EC}$, HSC, or $\mathrm{KC}$, establishes heterotypic cell-cell interactions and enables the detection of multicellular responses. For example, if hepatocellular injury in the human liver occurs secondary to cytokine release from either $\mathrm{HSC}$ or $\mathrm{KC}$, the hepatocellular injury response in the BHLT will likely require the concomitant presence and function of the HSC or $\mathrm{KC}$. A typical BHLT is designed to have substantial biomass, with overall size approximating $2.0-3.0 \mathrm{~mm}^{2} \times 0.5 \mathrm{~mm}$ and containing $\sim 1$ million cells ${ }^{8,13}$ and thus is compatible with molecular, biochemical, and histological endpoint measurements, although some modifications to these assays may be required to achieve optimal performance. BHLT create a substantial number of cell-cell interactions wherein the cells are in contact with each other without also being in direct contact with culture vessel plastics or liquid mediaboth of which can influence cell behavior due to mechanical stiffness or fluid dynamics. It is possible to examine BHLT histologically and detect complex histomorphological changes in response to various forms of injury within the spatial context of the tissue. For example, if a target biological response is identified and measured at the tissue level (i.e., vascular remodeling or the development of fatty liver disease), in vitro models that deliver histological data may be required. This may be particularly important for certain complex phenotypes and disease states, for which histological endpoints remain the gold standard of diagnosis and staging. In the case studies below, BHLT were employed in various applications and are assessed with respect to advantages and limitations. 
A

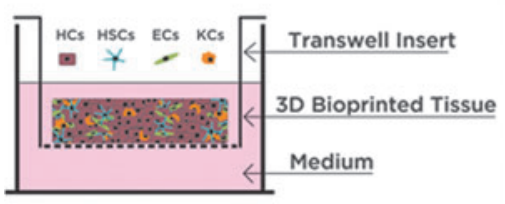

B

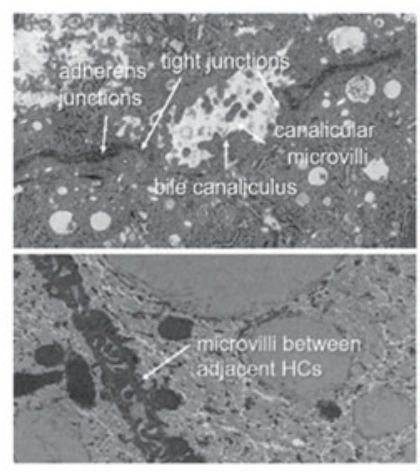

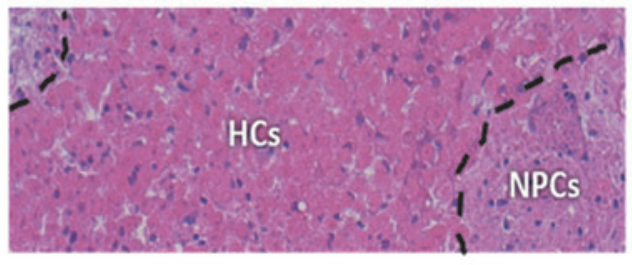

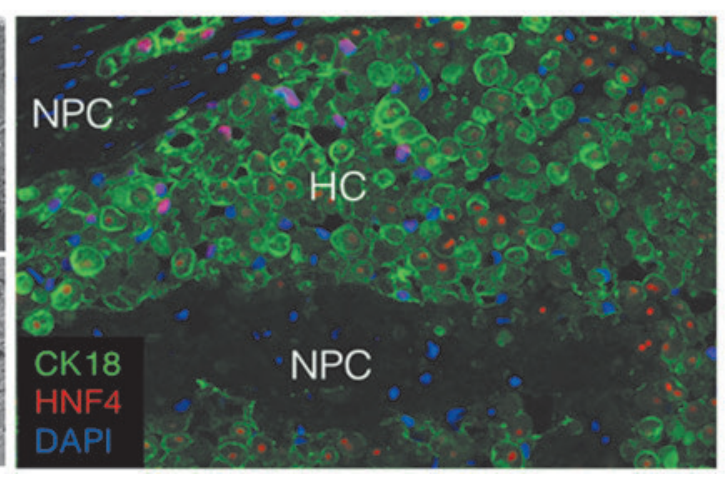

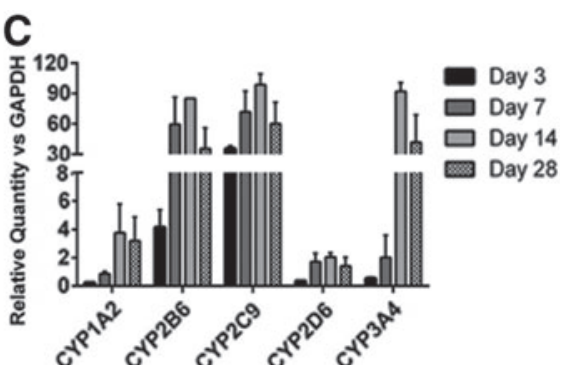

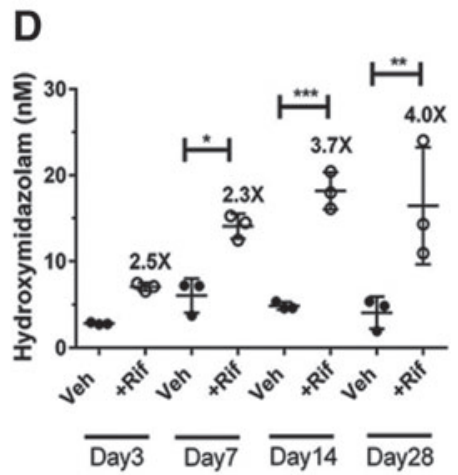

FIG. 1. Features of bioprinted human liver tissue. (A) BHLT (ExVive ${ }^{\circledR}$; Organovo, Inc.) are fabricated from HC, HSC, and EC using the Organovo MMX Bioprinter ${ }^{\mathrm{TM}}$ into the upper chamber of a standard 24-well TransWell ${ }^{\mathrm{TM}}$ plate (left panel). Spatially patterned, mature bioprinted liver organoids were fixed in $4 \%$ paraformaldehyde, paraffin-embedded, and subjected to $\mathrm{H} \& \mathrm{E}$ staining to visualize the tissue-like architecture and compartmentalization between the HC- and NPC-enriched regions (right panel). (B) TEM reveal focal bile canaliculi, tight junctions, and the presence of microvilli between adjacent HC. Immunohistochemical analysis highlights a $\mathrm{CK} 18^{+} / \mathrm{HNF}^{+}$field of $\mathrm{HC}$ surrounded by $\mathrm{CK} 18^{-} / \mathrm{HNF}^{-}$zones. (C) Quantitative RT-PCR analysis of selected CYP450 genes in BHLT demonstrates retention or gain of expression over 28 days postfabrication. Data were averaged from (three) independent replicates and expressed as RQ $\times 10,000$ after normalization to GAPDH, \pm SD. (D) Basal and rifampicin-induced CYP3A4 activity was assessed by the formation of $\alpha$-hydroxymidazolam and detection by mass spectrometry at the time points indicated. Data shown are the average of three replicates \pm SD. All statistics (one-way ANOVA) and outliers (Grubbs' test) were calculated using GraphPad Prism software. Significance: $* p<0.05, * * p<0.01, * * * p<0.001$. BHLT, bioprinted human liver tissues; EC, endothelial cells; H\&E, hematoxylin and eosin; HC, hepatocytes; HSC, hepatic stellate cells; NPC, nonparenchymal cell; RQ, relative quantity; RT-PCR, reverse transcription polymerase chain reaction; $\mathrm{SD}$, standard deviation; TEM, transmission electron micrographs.

\section{Case Study 1: Differential Toxicity of Tolcapone and Entacapone}

Parkinson's disease is a complex disorder, the pathogenesis of which is driven by multiple factors including genetic background, epigenetic changes, exposure to toxins, oxidative stress anomalies, neuroinflammatory reactions, hypoxicischemic conditions, metabolic deficiencies, and ubiquitinproteasome system dysfunction. ${ }^{22}$ Ultimately, patients develop neuropathological symptoms due to selective loss of dopaminergic neurons in the substantia nigra pars compacta and the deposition of Lewy bodies (abnormal deposits of alpha-synuclein) in the midbrain. In the 1960s, it was discovered that the administration of dopamine precursors (levodopa [L-DOPA]) provided symptomatic relief for many patients, but that repeated exposure led to a blunted response and a "wearing off" of the positive effects. ${ }^{23}$ Thus, adjunct treatments were developed aimed at perpetuating the effects of L-DOPA by either enhancing uptake or slowing its disposition through the use of dopamine agonists, monoamine oxidase inhibitors, and catechol- $O$-methyltransferase (COMT) inhibitors. COMT inhibitors, such as tolcapone and entacapone, inhibit degradation of L-DOPA resulting in sustained plasma levels and enhanced central dopaminergic activity. Tolcapone was suspended from use and subsequently issued a black box warning for potential liver toxicity due to multiple cases of drug-induced fulminant hepatitis, several of which were fatal. Tolcapone is believed to exert its toxic effects by activating the inflammasome and by uncoupling oxidative phosphorylation in mitochondria and reducing the capacity for ATP production. $^{24,25}$ Additionally, variability in individuals' metabolic profiles may lead to higher plasma levels (and thus greater risk of toxicity) for some patients. ${ }^{24}$ In contrast, the structurally similar drug entacapone has not caused serious hepatotoxicity in patients and appears to lack the toxic mechanisms associated with tolcapone. ${ }^{26}$

In vitro comparative studies were conducted with tolcapone and entacapone using a BHLT model, ExVive ${ }^{\circledR}$ (Organovo, Inc.), at concentrations approximating $2.4 \times(50 \mu \mathrm{M})$ and $9.5 \times(200 \mu \mathrm{M})$ Cmax for tolcapone (https://www.accessdata .fda.gov/drugsatfda_docs/label/2013/020697s004lbl.pdf) and $2.5 \times(10 \mu \mathrm{M})$ and $10 \times(40 \mu \mathrm{M}) \mathrm{Cmax}$ for entacapone (https:// www.drugs.com/pro/entacapone.html). As anticipated from clinical data, tolcapone caused a significant decrease in ATP production at both concentrations, a spike in alanine aminotransferase (ALT) at $2.4 \times \mathrm{Cmax}$, and significant loss of detectable ALT at $9.5 \times \mathrm{Cmax}$, suggesting both mitochondrial damage and hepatocellular injury (Fig. 2A, 

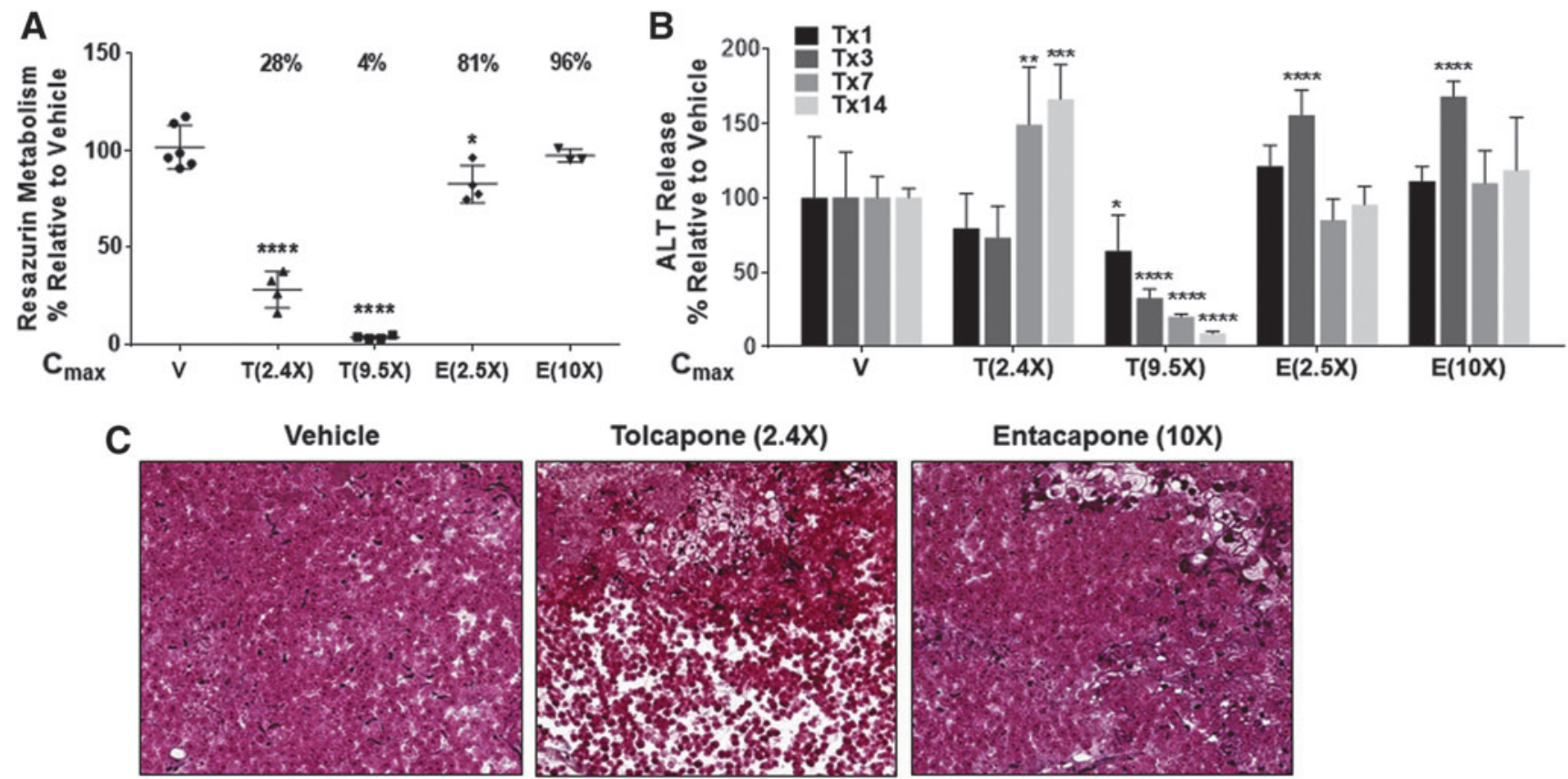

Tolcapone (2.4X)
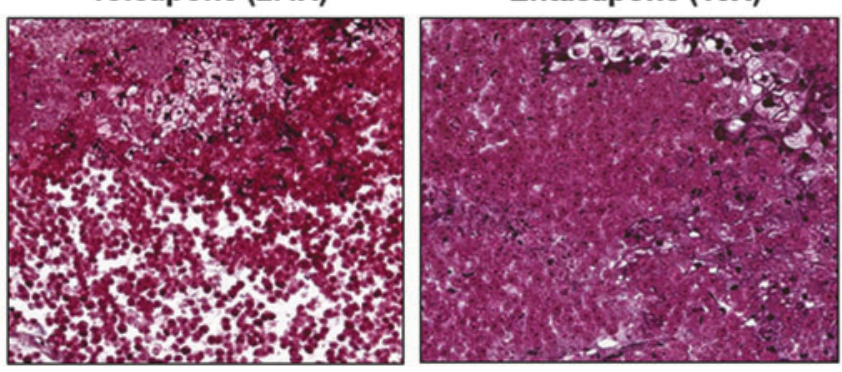

FIG. 2. Use Case Study 1: Biochemical and histological detection of compound toxicity. Tolcapone and entacapone were administered to BHLT (ExVive) daily for 14 days. (A) Resazurin metabolism in tissue at day 14 suggests significant tissue injury in response to tolcapone at $2.4 \times$ or $9.5 \times \mathrm{Cmax}$, whereas entacapone exposure at $2.5 \times$ and $10 \times \mathrm{Cmax}$ did not (one-way ANOVA with Tukey's multiple comparisons). (B) ALT was measured in the culture supernatant at days 1, 3, 7, and 14 of compound exposure. At $2.4 \times \mathrm{Cmax}$, tolcapone-exposed tissues exhibited significantly elevated ALT at days 7 and 14 , whereas ALT at $9.5 \times$ Cmax fell well below the levels of the vehicle group (two-way ANOVA with Tukey's multiple comparisons). (C) FFPE BHLT were sectioned and stained with H\&E. At day 14, tolcapone-treated BHLT (2.4 $\times$ Cmax) exhibited clear signs of tissue damage, including dissociation of adjacent hepatocytes, cell shrinkage, and cell loss. In contrast, entacapone-treated tissues resembled vehicle, even at the highest concentration $(10 \times \mathrm{Cmax})$. Significance: $* p<0.05$, $* * p<0.01, * * * p<0.001, * * * * p<0.0001$. ALT, alanine aminotransferase; FFPE, formalin-fixed paraffin-embedded.

B). In contrast, entacapone did not adversely affect ATP levels, although mild transient elevations in ALT were noted at early time points of exposure. Moreover, histological analyses of the vehicle- and drug-treated tissues revealed clear signs of hepatocellular injury and cell loss in the tolcapone-treated tissues (Fig. 2C), confirming the biochemical observations.

While the histological observations of tolcapone toxicity are unique to BHLT, other in vitro model systems have detected tolcapone toxicity and differentiated its effects from entacapone, including HepG2 cell cultures, ${ }^{27}$ micropatterned cocultures, ${ }^{28}$ and an in silico mechanistic model (DILIsym ${ }^{\circledR}$ ). ${ }^{26}$ BHLT are competent for the basic assessment of hepatocellular injury in both the acute and chronic exposure settings and have been employed successfully in the assessment of toxicity response to additional compounds, including trovafloxacin and levofloxacin, ${ }^{13}$ acetaminophen, ${ }^{29,30}$ methotrexate (MTX) ${ }^{14}$ (Norona et al., "Bioprinted human liver provides insight into the role of Kupffer cells in modulating early events in TGFB1 and methotrexate-induced fibrotic injury," submitted), benzbromarone, danazol, tamoxifen, and perhexiline. ${ }^{31}$ Due to complexity and cost, BHLT may not displace simpler higher throughput in vitro systems for routine acute toxicity screening; however, they may be particularly useful in the safety assessment of compounds that cause tissue damage through multicellular mechanisms or rely in part on histological endpoints for detection and interpretation. Subtle effects, such as disruption of intercellular junctions, and loss of cell- cell adhesion, such as those noted with tolcapone exposure, may be detected histologically in advance of significant biochemical evidence of overt toxicity. Techniques such as in situ hybridization and spatial transcriptomics make it possible to investigate signal transduction and mechanism at the molecular level within histological sections of the BHLT, so that specific toxicity-associated outcomes can be interpreted within the spatial context of the tissue and related cell-cell interactions. With the growing epidemics of obesity, type 2 diabetes mellitus, and nonalcoholic fatty liver diseases (NAFLD) in the developed world, it may grow increasingly more important to the safety of most drugs in models that approximate these susceptible patient populations.

\section{Case Study 2: Monocrotaline-Induced Tissue Injury}

Pyrrolizidine alkaloids (PAs), such as monocrotaline (MCT), are found in $>6000$ species of plants within the Asteraceae, Boraginaceae, Compositae, and Fabaceae families, many of which are used in the preparation of herbal teas and remedies and may contaminate dietary supplements, herbal products, and honey. Ingestion of PAs causes dose-dependent toxicity to multiple organ systems, including the lung, kidney, and liver. In addition to isolated reports of human exposure, rodent models have been developed to enable mechanistic investigations into MCT toxicity. Hepatotoxicity requires metabolic activation of MCT by CYP3A4 to a toxic pyrrole intermediate, which leads to a spectrum of changes including the release of proinflammatory 
cytokines, production of fibrogenic transforming growth factor beta $(\mathrm{TGF} \beta)$ and activation of HSC, formation of protein and DNA adducts, and endothelial cell damage and hepatocellular injury. ${ }^{32-35}$ Microscopically, hepatic changes are characterized mainly by centrilobular necrosis and injury to sinusoidal EC. ${ }^{32-35}$ Accordingly, attempts to recapitulate MCT toxicity in vitro require a system capable of metabolic activation (i.e., CYP3A4-competent hepatocytes), cytokine production, HSC capable of mounting a response to TGF $\beta$, and EC.

BHLT were exposed daily for 2 weeks to $20 \mu \mathrm{M}, 200 \mu \mathrm{M}$, and $2 \mathrm{mM}$ MCT and assessed for cytokine production, hepatocyte synthetic function (albumin secretion), general tissue viability (ATP production), and histological evidence of vascular abnormalities, hepatocyte injury, and stellate cell activation. $^{36}$ Although ATP production was not significantly altered at any concentration of MCT (Fig. 3A), a decrease in albumin synthesis in treated tissues over time was suggestive of mild hepatocellular injury (Fig. 3B). While histological examination showed little evidence of overt hepatocellular toxicity, MCT exposure caused extensive injury and remodeling of the nascent microvascular structures within the tissues with the formation of large, branched, hypertrophic vascular lesions (Fig. 3D). Interestingly, smooth muscle actin $(\mathrm{SMA})^{+}$cells presumed to be activated HSC were found throughout MCT-treated tissues and localized in close proximity to the hypertrophic $\mathrm{CD} 31^{+}$endothelial vascular structures, circumstantially supporting the hypothesis that activated stellate cells serve as pericytes and facilitate angiogenesis during liver fibrosis. ${ }^{37-39}$ The abundance of secreted interleukin (IL)- 8 and IL- $1 \beta$ in the media surrounding the tissue suggests that inflammation was mildly increased at the highest MCT concentration (Fig. 3C). The source of these cytokines was presumed to be HSC and/or $\mathrm{EC}$, as $\mathrm{KC}$ were not a component of these tissues.

The direct hepatocellular toxicity of MCT has been detected in vitro using chicken hepatocytes, ${ }^{40}$ a HepG2/ $\mathrm{C} 3 \mathrm{~A}$ rat liver $\mathrm{S} 9$ fraction co-incubation system, ${ }^{41}$ and precision-cut rat liver slices. ${ }^{42}$ MCT-induced glutathione depletion and cytotoxicity were also observed in isolated murine liver EC. ${ }^{43}$ Outside of in vivo rodent models, there has not been a model system to date other than the BHLT that concomitantly detects the vascular injury, hepatocellular injury, inflammatory cytokine production, and HSC activation that occur in response to MCT. None of these cellular responses alone (i.e., hepatocyte damage, stellate cell activation, or stimulation of EC) could recapitulate the full spectrum of outcomes associated with MCT toxicity; threedimensionality, multicellularity, and the existence of biologically relevant cell-cell interactions between the HSC, EC, and hepatocytes were necessary. Perhaps one of the most compelling observations in the BHLT model is the apparent deactivated state of the HSC within the tissues, particularly in stellate cells that do not come in direct contact with plastic or media surfaces. The establishment of a deactivated or
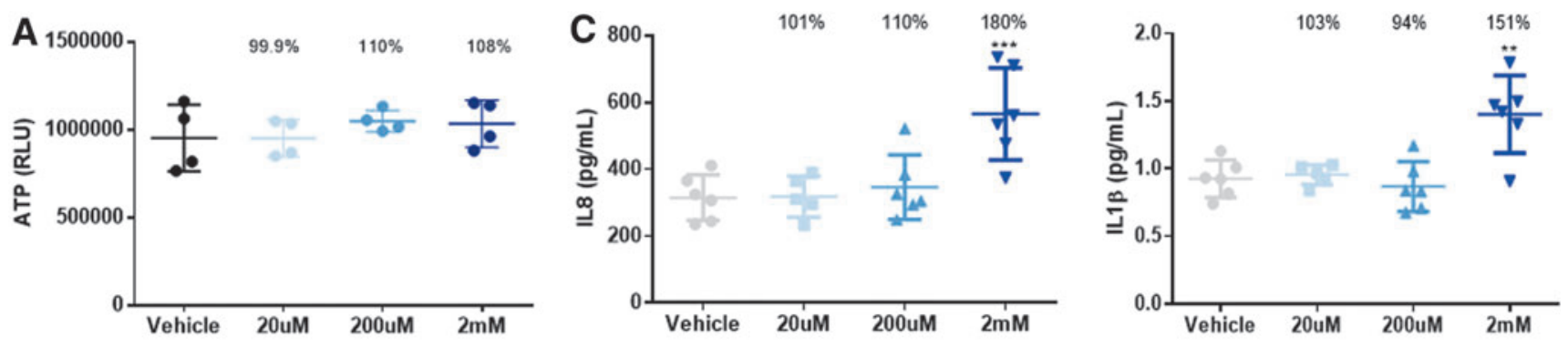

B

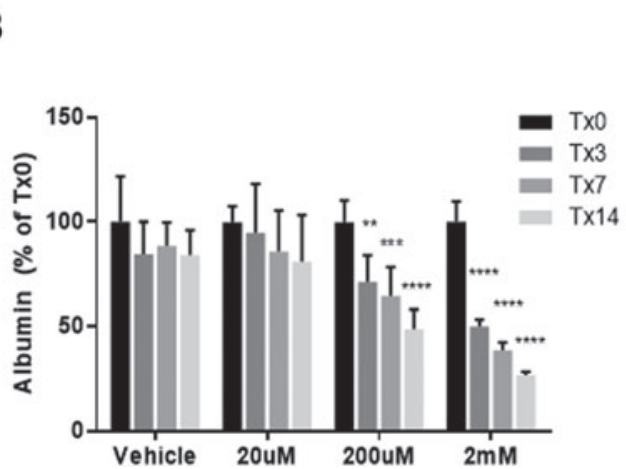

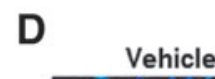

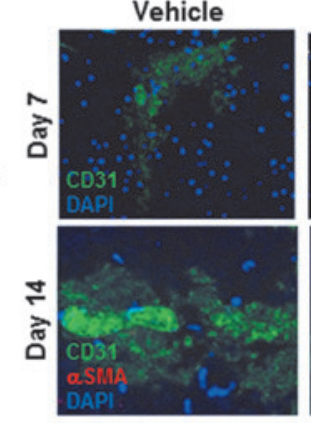

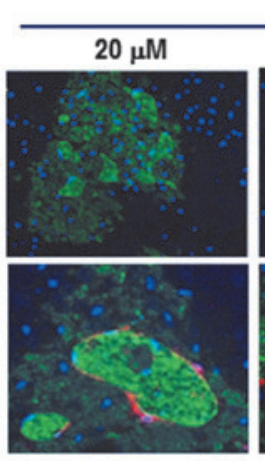

Monocrotaline

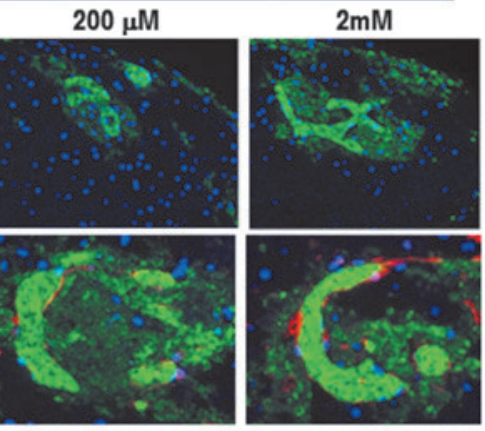

FIG. 3. Use Case Study 2: Complex multicellular compound toxicity. Monocrotaline was administered to BHLT (ExVive) daily at $20 \mu \mathrm{M}, 200 \mu \mathrm{M}$, and $2 \mathrm{mM}$ concentrations and compared with vehicle control after 7- and 14-day exposures. (A) ATP levels in tissue lysates were not significantly altered after 14 days of exposure to monocrotaline, suggesting minimal overt tissue toxicity. (B) Albumin levels in the culture supernatant declined over time in the $200 \mu \mathrm{M}$ and $2 \mathrm{mM}$ treatment groups, suggesting compromised hepatocellular function at higher concentrations (two-way ANOVA with Dunnett's multiple comparisons). (C) IL-8 and IL-1 $\beta$ were quantified on day 7 in the culture supernatants with a multiplexed cytokine assay (MSD proinflammatory 10-spot assay) and found to be elevated in the $2 \mathrm{mM}$ treatment group compared with vehicle (one-way ANOVA with Dunnett's multiple comparisons). Significance: $* * p<0.01, * * * p<0.001, * * * * p<0.0001$. (D) Immunohistochemical analyses of FFPE BHLT revealed a dose-dependent extensive remodeling of CD31 ${ }^{+}$EC evident by day 7 post-treatment (top panel). By day 14, numerous hypertrophic vascular structures $\left(\mathrm{CD} 31^{+}\right)$were found throughout monocrotaline-exposed tissues in close association with $\alpha \mathrm{SMA}^{+}$myofibroblast-like cells. IL, interleukin; SMA, smooth muscle actin. 
quiescent-like state in the stellate cells of BHLT subsequently enables the clear detection of stellate cell activation upon stimulus with MCT or other activation factors-a phenomenon that has proven difficult to capture in traditional two-dimensional (2D) or monoculture systems. While a simple homogeneous cell culture can respond to a stimulus with what could be considered a first-order response, more complex second- and third-order responses may be obtained with the additional cell types and three-dimensionality. This example highlights the suitability of BHLT for applications in which the complex tissue level response, versus the simple cellular level response, is critical to interpretation of outcomes and evaluation of mechanisms.

\section{Case Study 3: Modeling Progressive Liver Fibrogenesis with TGF $\beta$}

Fibrosis is a common conserved wound healing response that occurs in numerous tissues, including the liver, secondary to a persistent injury or disease. There is a need to better understand the molecular and cellular mechanisms underlying liver fibrosis and how they might vary based on the mode of injury. ${ }^{44} \mathrm{~A}$ number of precipitating factors leading to fibrotic injury have been gleaned from animal models, but there are numerous challenges associated with the translation of these outcomes to humans. Species differences in drug-metabolizing enzymes, transporters, hepatic drug deposition, immune response, and temporal aspects of the response may compromise translation between preclinical animal models and the clinic. ${ }^{45,46}$ Compound-induced fibrotic injury in humans is commonly associated with prolonged treatment of chronic disorders, such as rheumatoid arthritis and psoriasis with drugs like MTX. ${ }^{47,48}$ Given the persistent challenges associated with detecting and evaluating liver fibrosis in humans as well as the limited translatability of animal models, there have been considerable efforts to develop and adopt more human relevant alternative testing and screening strategies that do not rely heavily on animal toxicity data. ${ }^{99,50}$

In 2016, Norona et al. reported outcomes from subacute exposure of BHLT (ExVive) to known profibrotic agents MTX, thioacetamide (TAA), or TGF $\beta{ }^{14}$ Treatment with TGF $\beta 1$ activated HSC and yielded moderate fibrotic change in the BHLT with little evidence of hepatocellular damage. However, concentration- and time-dependent elevations of lactate dehydrogenase were observed for both MTX and TAA and were accompanied by tissue remodeling, NPC migration/activation, histological evidence of collagen deposition, transient elevations in proinflammatory, immunomodulatory, and chemotactic cytokines, and the expression of ACTA2 and COL1A1. These initial studies provide compelling evidence of mild injury profiles over a 2-week time frame with the acquisition of robust fibrogenic processes at a tissue, cellular, and molecular level.

In follow-up experiments, BHLT containing $\mathrm{KC}$ were exposed to MTX or TGF $\beta$, and again, a progressive fibrogenic phenotype was established in the BHLT in response to injury (shown after 2 weeks of exposure in Fig. 4A) (Norona et al., "Bioprinted human liver provides insight into the role of Kupffer cells in modulating early events in TGFB1 and methotrexate-induced fibrotic injury," submitted). Microarray analysis of vehicle-treated versus TGF $\beta$-treated BHLT revealed the modulation of key pathways including upregula- tion of extracellular matrix (ECM) remodeling genes known to be involved in liver injury with fibrosis (Fig. 4B). Crosscomparison of BHLT tissue data with differentially expressed genes from an independent study of human healthy control liver versus confirmed NAFLD/nonalcoholic steatohepatitis (NASH) disease specimens revealed significant overlap in modulated pathways (Fig. 4C, D). Taken together, these data suggest that BHLT undergoing chronic injury with fibrosis recapitulate some key molecular and phenotypic features of human liver fibrosis and may thus serve as in vitro human models for interventional studies.

Recently, further studies were conducted with TGF $\beta$-treated BHLT, wherein a TGF $\beta$ receptor type I kinase inhibitor (galunisertib) was introduced either concomitant with TGF $\beta$ treatment (data not shown) or after 7 days of TGF $\beta$ treatment (Fig. 5A). ${ }^{15}$ When galunisertib was introduced after TGF $\beta$ induced fibrogenesis was initiated, the drug reduced and resolved fibrotic injury despite continued exposure to TGF $\beta$ (Fig. 5B). As anticipated, treatment with galunisertib demonstrated blockade of canonical TGF $\beta$ signaling, evidenced by pSMAD $2 / 3$, and inhibited the TGF $\beta$-induced secretion of TIMP- $1 .{ }^{15}$ Furthermore, the drug effected significant reductions in collagen deposition, hyaluronic acid, and fibronectin (Fig. 5C). Collectively, these biochemical and histological endpoints provide proof of concept that active fibrogenesis can be established and modulated in vitro in BHLT.

Aspects of fibrogenic liver injury have been reported in other in vitro model systems, including TGF $\beta$-treated spheroids composed of HepG2 cells, human telomerase reverse transcriptase (hTERT)-immortalized human HSC, and monocytederived macrophages, wherein fibrosis-associated genes were upregulated and collagen 1 was detectable by immunohistochemistry. ${ }^{51}$ Additional studies demonstrated similar outcomes in allyl alcohol- or MTX-treated spheroids generated from HepG2 cells and primary human HSC. ${ }^{52}$ Culture- or TGF $\beta$-activated rodent or human HSC are also used to test the ability of a compound to exacerbate or inhibit activationassociated endpoints, such as profibrotic gene expression or collagen secretion, but this strategy detects HSC effects only outside of the context of other cell types or tissue architecture. There is much to be gained by understanding HSC activation, migration, and ECM deposition within the context of spatially arranged 3D tissues and specific cell-cell interactions. For example, it is highly likely that the $\alpha \mathrm{SMA}(+)$ HSC localized within thick bands of collagen and adjacent to hepatocytes that exhibit signs of toxic injury will have different gene expression patterns and secreted molecule profiles when compared with the $\alpha \mathrm{SMA}(-) \mathrm{HSC}$ located away from sites of active fibrogenesis. BHLT uniquely provide a means to establish an advanced state of fibrosis in human liver tissue, wherein trichrome-detectable fibrillar collagens are present and multiple gene, protein, and cytokine signals associated with clinical disease are represented. Many in vitro systems are too short-lived to support the temporal progression of HSC activation and collagen deposition or they lack a sufficient quantity of "deactivated" or "quiescent-like" HSC and thus cannot further respond to activation stimuli. The demonstration of compound-induced modulation of established fibrosis provides proof of concept that BHLT have utility not only in the safety assessment of drugs that may cause fibrotic injury but also in the search for treatments that reduce or resolve the liver damage. 


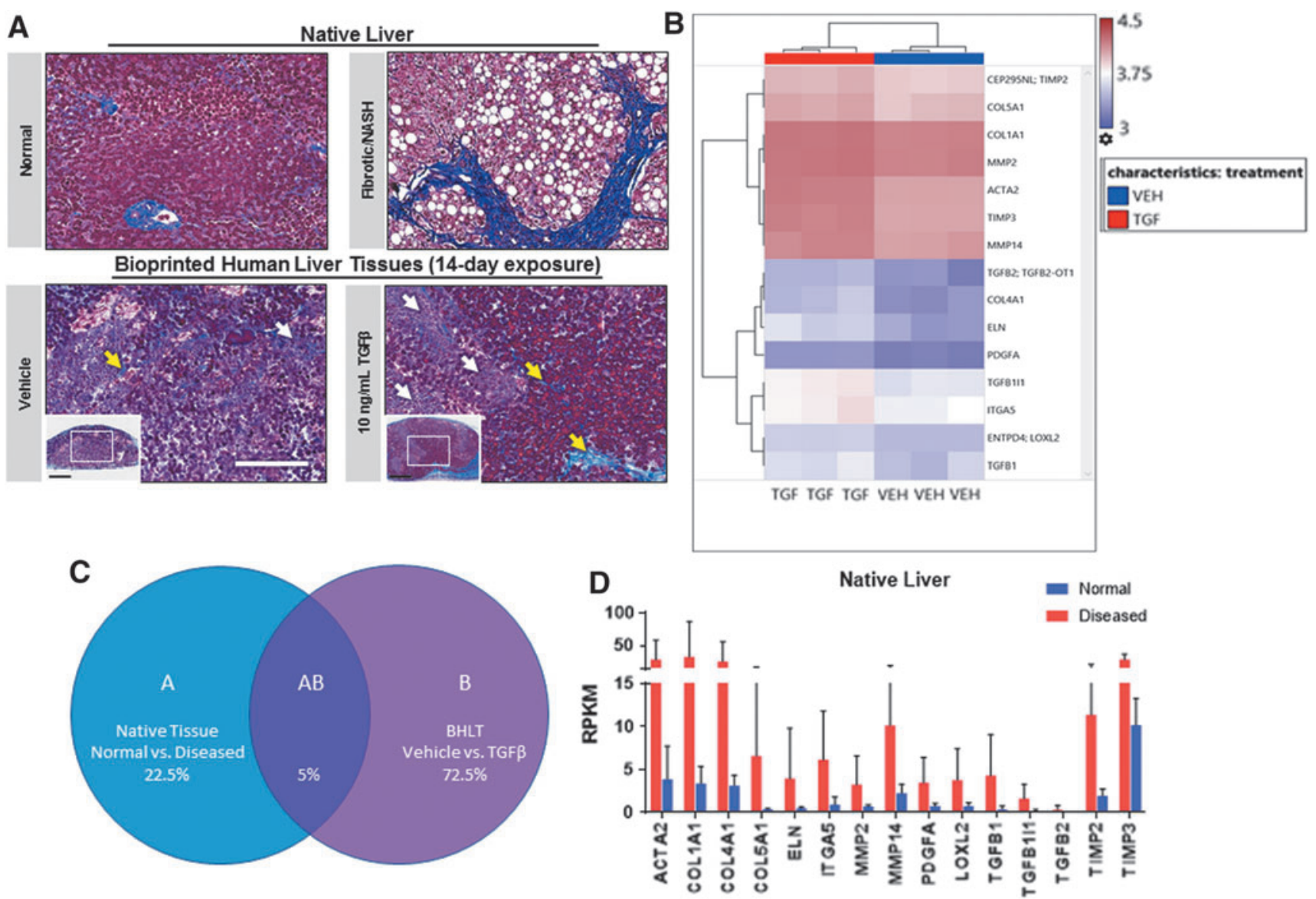

FIG. 4. Use Case Study 3: Tissue response to profibrotic agents. (A) Representative photomicrographs (Gomori's trichrome stain) are shown from normal native human liver tissue and from a patient with confirmed NASH and fibrosis. The lower panel shows BHLT (ExVive) with KC that have been treated daily with vehicle or $10 \mathrm{ng} / \mathrm{mL} \mathrm{TGF} \beta$ for 14 days. Mature collagen deposition appears as bright blue-stained fibrils (yellow arrows) and diffuse areas of collagen (white arrows). Scale bar $=300 \mu \mathrm{m}$; inset scale bar $=75 \mu \mathrm{m}$. (B) The BHLT treated with TGF $\beta$ as represented in (A) were subjected to microarray-based gene expression analysis. Differential expression was determined by FDR of $p<0.05$ and absolute fold change $>1.5$. Hierarchical clustering representation (log2 transformation) of selected differentially expressed genes from vehicle versus TGF $\beta$-treated BHLT $(n=3)$ are shown (TAC 4.0 software). (C) In separate experiments, RNAseq and differential gene expression analysis (Gubra, DESeq2 software) was performed with (five) healthy human liver tissue samples and (five) human liver tissue samples with confirmed NASH/fibrosis to identify significantly differentially expressed genes among diseased versus normal samples $(p<0.05)$. Cross-comparison of both groups of significant differentially expressed genes revealed 5\% overlap between both sets of experiments. (D) Selected differentially expressed genes from the native tissue analysis are graphed as RPKM. Venn diagram gene list comparison was performed using TAC 4.0 software (ThermoFisher). FDR, false discovery rate; KC, Kupffer cells; NASH, nonalcoholic steatohepatitis; RPKM, reads per kilobase million; TAC, Transcriptome Analysis Console; TGF $\beta$, transforming growth factor beta.

\section{Case Study 4: Modeling NAFLD/NASH-Like Phenotypes Secondary to Diet-Induced Injury}

The most prevalent forms of liver injury leading to fibrosis in the developed world are chronic Hepatitis $\mathrm{C}$ virus (HCV) infection, alcohol abuse, and NASH. ${ }^{53}$ The growing epidemics of metabolic disorders, including obesity and type II diabetes, contribute heavily to the increasing incidence of fatty liver disease and NASH. The liver has a remarkable capacity for regeneration and compensation and is so adept at making up for lost function that the early stages of NAFLD and NASH often go undetected clinically. Diagnosis often comes late in progression of the disease when the liver can no longer compensate (decompensated NASH) and patients are left with severely compromised liver function and few treatment op- tions. ${ }^{53}$ Although end-stage liver fibrosis is characterized by some common histological and molecular features, it is probable that the mechanisms driving the progression of virus-, alcohol-, drug injury-, and NASH-induced fibrosis are partially distinct. Therefore, the interventional strategies that apply well to one etiological basis of injury may fall short in others. As animal and in vitro models of progressive liver fibrosis are needed to enable drug discovery and guide the clinical approach to the treatment of disease, it may be important to consider not only the end result (fibrosis) but also the specific initiating and perpetuating events that produced the fibrosis.

The discovery and development of in vitro models of NAFLD and NASH is an active and high-profile area of investigation by academe, pharma, and biotech sectors. Experiments have been conducted to determine whether BHLT can 

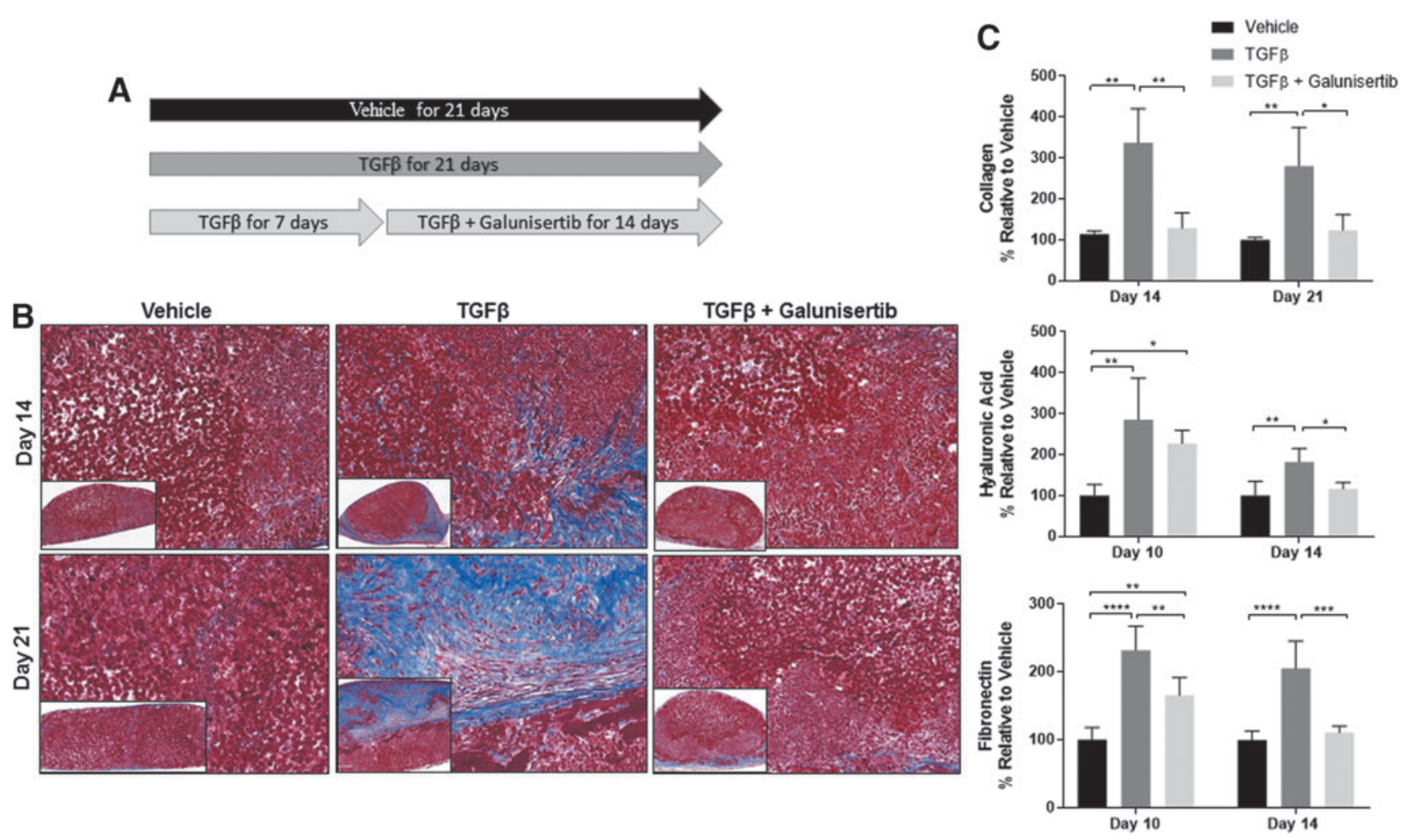

FIG. 5. Use Case Study 3: Modulation of fibrogenesis with ALK5 inhibitor. BHLT (ExVive) were treated for a total of 21 days with vehicle, $3.3 \mathrm{ng} / \mathrm{mL}$ TGF $\beta$, or $3.3 \mathrm{ng} / \mathrm{mL}$ TGF $\beta$ followed by cotreatment with $3.3 \mathrm{ng} / \mathrm{mL}$ TGF $\beta+5.0 \mu \mathrm{M}$ galunisertib as depicted in (A). (B) Tissues were FFPE on days 14 and 21 and subjected to trichrome staining to reveal collagen deposits (bright blue). Insets are low magnification images showing distribution of collagen throughout tissue. (C) Adjacent histological sections ( $15 \mu \mathrm{m}$ thick) were subjected to picrosirius red/fast green assays and quantification of the dye by spectrophotometry $(n=3)$. Hyaluronic acid and fibronectin were quantified in culture supernatants by ELISA. $n=5$ (day 10) and $n=4$ (day 14). Significance by two-way ANOVA with Sidak's multiple comparisons: $* p<0.05, * * p<0.01, * * * p<0.001, * * * * p<0.0001$.

be driven to develop key features of NAFLD and NASH (i.e., inflammation, steatosis, hepatocytes with ballooning degeneration, and histologically evident fibrosis) by simply exposing the tissues to high levels of sugar and fatty acids in the media to mimic the typical high-fat high-sugar diet associated with obesity. ${ }^{11}$ Interestingly, multiple combinations of glucose, fructose, saturated and unsaturated free fatty acids (FFAs), and inflammatory stimuli such as lipopolysaccharide or IL$1 \beta$ have demonstrated the capacity to evoke a tissue response reminiscent of NAFLD/NASH with fibrosis in BHLT (Fig. 6A-C). ${ }^{11,12}$ Key human-relevant histological features of NAFLD/NASH are detected, including macrovesicular and microvesicular steatosis, ballooning degeneration, and fibrosis. Both $\mathrm{KC}$ and activated HSC play key roles in the development of a robust NASH-like phenotype, driving inflammation and fibrosis, respectively (Fig. 6E, F).

Given the emphasis on NASH and the strong interest in human models that can replicate key disease-relevant features, there are significant efforts under way to develop and validate in vitro models to complement the rodent preclinical models. Studies conducted with micropatterned co-cultures comprising primary human hepatocytes, 3T3-J2 murine embryonic fibroblasts, and primary human HSC demonstrated that increasing the number of HSC or the application of HSC-conditioned media caused hepatocellular steatosis and some evidence of profibrotic pathway activation, which were inhibited by FXR (farnesoid $\mathrm{X}$ receptor) activation and NADPH oxidase inhibi- tion. ${ }^{54}$ A perfused model of liver (LiverChip ${ }^{\circledR} ; \mathrm{CN}$ Bio Innovations, United Kingdom) was cultured for 14 days in standard media or media containing a high concentration of FFAs. Hepatocytes in the high FFA media accumulated lipids as detected by Oil Red O staining, exhibited loss of metabolic functions, and upregulated genes and adipokines associated with fatty liver disease. ${ }^{55} \mathrm{~A}$ perfused liver model consisting of a collagen-embedded hepatocyte layer, a porous membrane, and a layer of macrophages and HSC (Hemoshear, Charlottesville, VA) was cultured in the presence of glucose, insulin, and FFAs to induce lipid accumulation and produce a lipidomic and transcriptomic profile that correlated with NASH clinical samples. ${ }^{56}$ In addition to TGF $\beta$ secretion, the exposure to the NASH induction conditions caused modulation of genes associated with fibrogenesis (stellate activation, ECM deposition). ${ }^{56}$

Despite the ongoing efforts toward validating and adopting biomarkers for the diagnosis and staging of NAFLD and NASH, biopsy and histopathological assessment remains the gold standard. ${ }^{57}$ Confirmation of NASH requires observation of key features, including steatosis, hepatocellular ballooning, and the accumulation of fibrillar collagen. Once the disease phenotype has been established, biochemical and molecular endpoint measurements can be interpreted safely. Due to the complex multifactorial nature of NAFLD and NASH, the observation of a single phenotypic feature or detection of biochemical and molecular markers may not be sufficient for establishing the disease state or assessing modulation. 
A
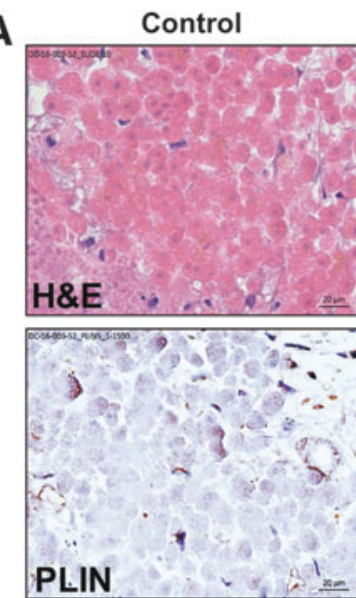

B

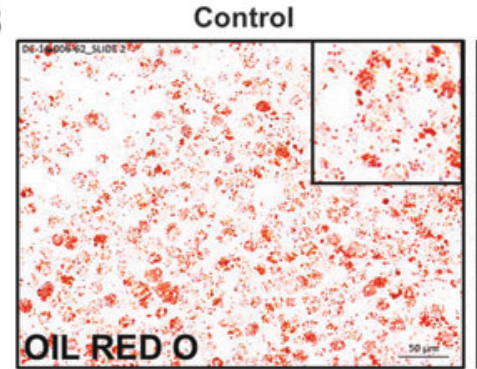

+ FFA [low]
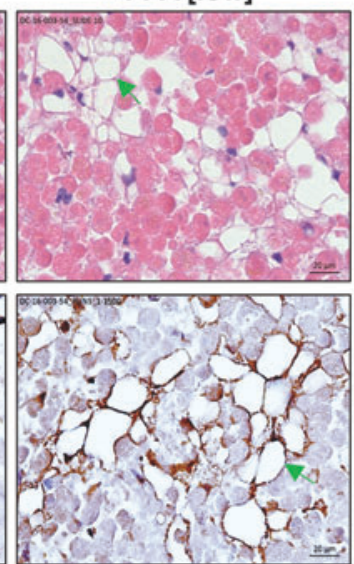

+ FFA [high]
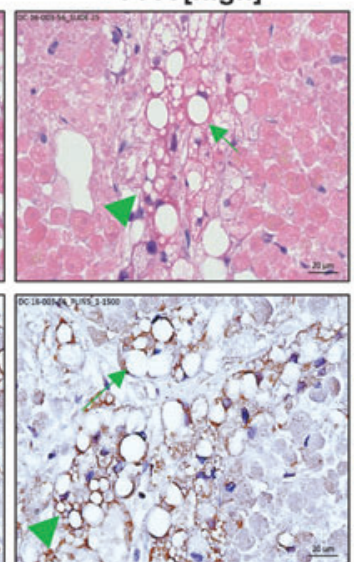
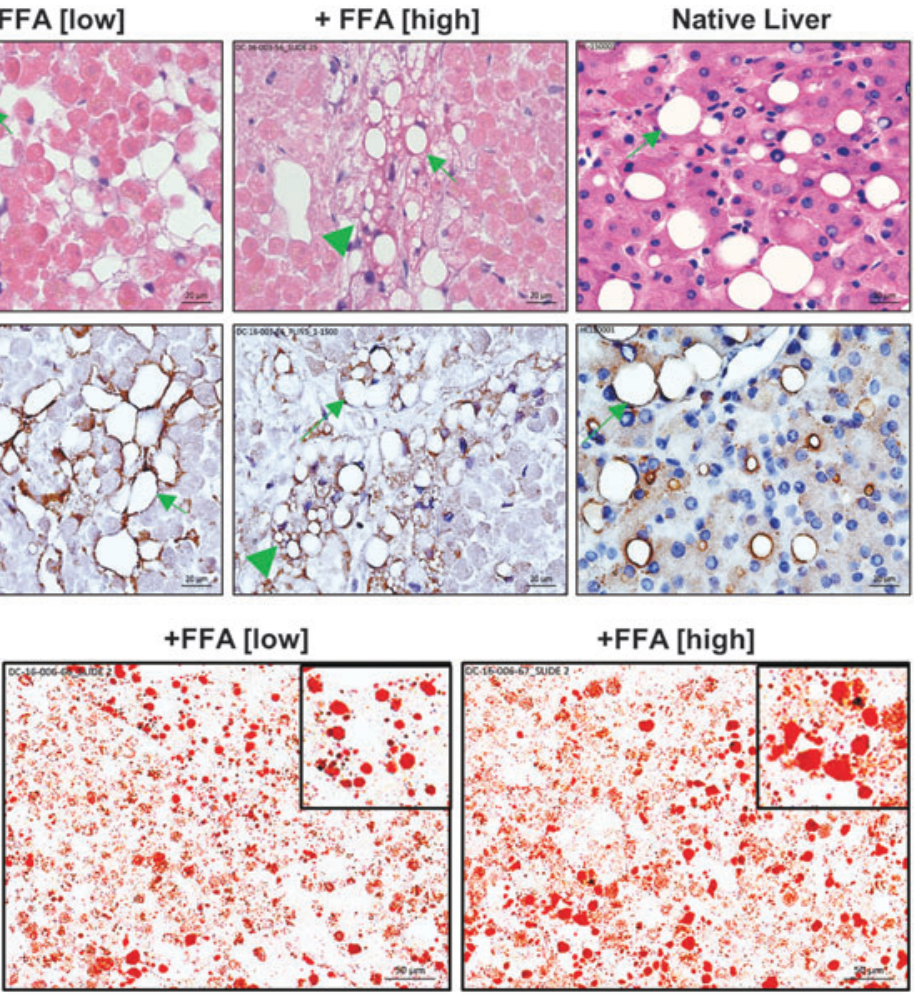

C
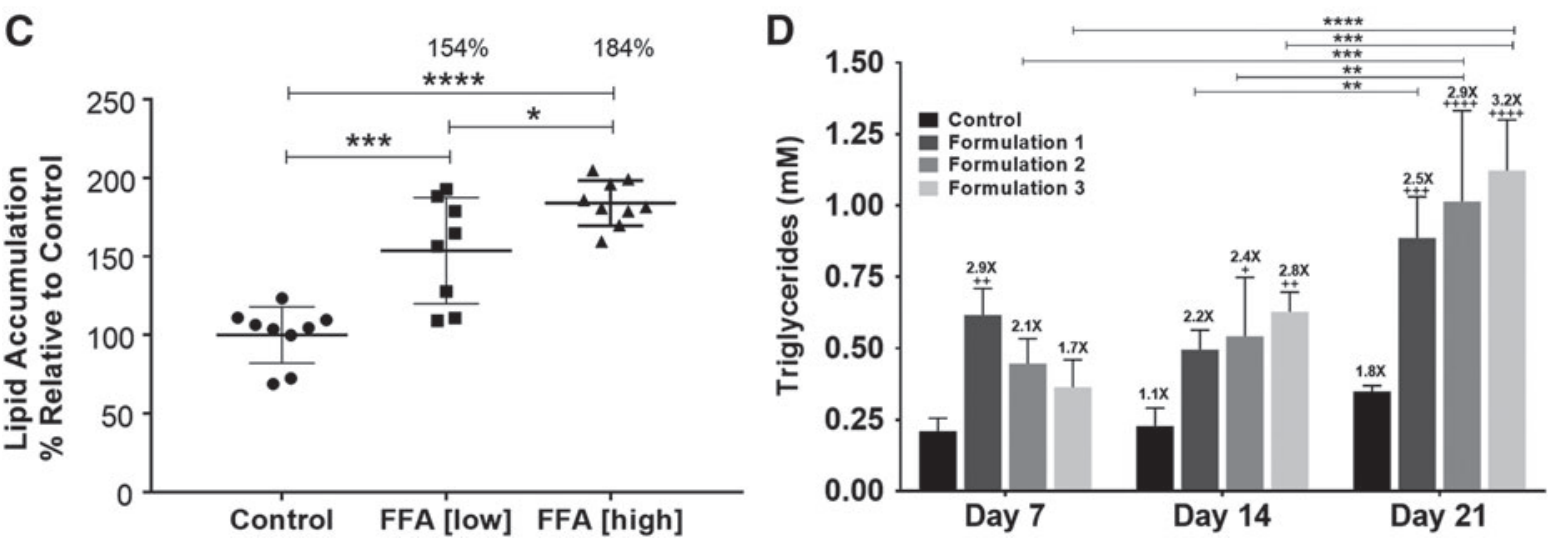

FIG. 6. Use Case 4: Diet-induced liver damage. (A) BHLT (ExVive) containing Kupffer Cells (KC) were fed daily for 21 days with media containing free-fatty acids (FFAs) to induce lipid droplet formation. H\&E staining of FFA-treated tissue shows both macro- (arrows) and micro- (arrowheads) vesicular phenotypes (B) Frozen sections from control and FFA-fed BHLT were stained with Oil Red O to identify lipid droplets. (C) Quantification of Oil Red O in tissue sections by ImageJ. Significance determined via one-way ANOVA with Tukey's multiple comparisons test. ${ }^{*} p<0.05,{ }^{*} p<0.01,{ }^{*} * *<<0.001$, $* * * * p 0.0001$. (D) Triglyceride (TG) quantification in BHLT after exposure to (3) blended formulations of FFA and high sugars. Significance determined via two-way ANOVA with Tukey's multiple comparisons test. $* * p<0.01, * * * p<0.001$, **** $p<0.0001$; Bar labels are fold change above time-matched controls. Significance determined via two-way ANOVA with Dunnett's multiple comparisons test. ${ }^{+} p<0.05,{ }^{++} p<0.01,{ }^{++} p<0.001,{ }^{++++} p<0.0001$. (E) Top panel: BHLT with KC were stimulated with LPS and exposed to FFA for 21 days and compared to native human liver tissue with confirmed NASH. Desmin+(green) and $\alpha$-SMA+(red) cells were labeled by immunohistochemistry. Bottom panel: trichrome stained tissues highlight the well-developed fibrosis in LPS/FFA-treated and native NASH tissues (F; regions of fibrotic collagen staining in blue) and steatosis (green arrows), similar to native tissue samples. (F) Both TNF $\alpha$ and IL1 $\beta$ were increased in the culture supernatant in the presence of KC, LPS, and FFAs. Significance determined via one-way ANOVA with Tukey's multiple comparisons test. * $p<0.05$, $* * p<0.01, * * * p<0.001,{ }^{* * * *} p<0.0001$. FFA, free-fatty acid; LPS, lipopolysaccharide; TG, triglyceride.

BHLT are ideally suited for the investigation of compound effects at the tissue level in the context of an NAFLD or NASH phenotype, due to their ability to correlate the disease-defining phenotypic features with modulation of specific targets at the biochemical and molecular levels. While initial efforts have been focused on developing and validating BHLT for use in drug development for the treatment of NAFLD and NASH, it may be compelling to examine the safety of drugs-especially those targeted to patient populations at high risk for NASH-in BHLT with confirmed NASH phenotype. 
E
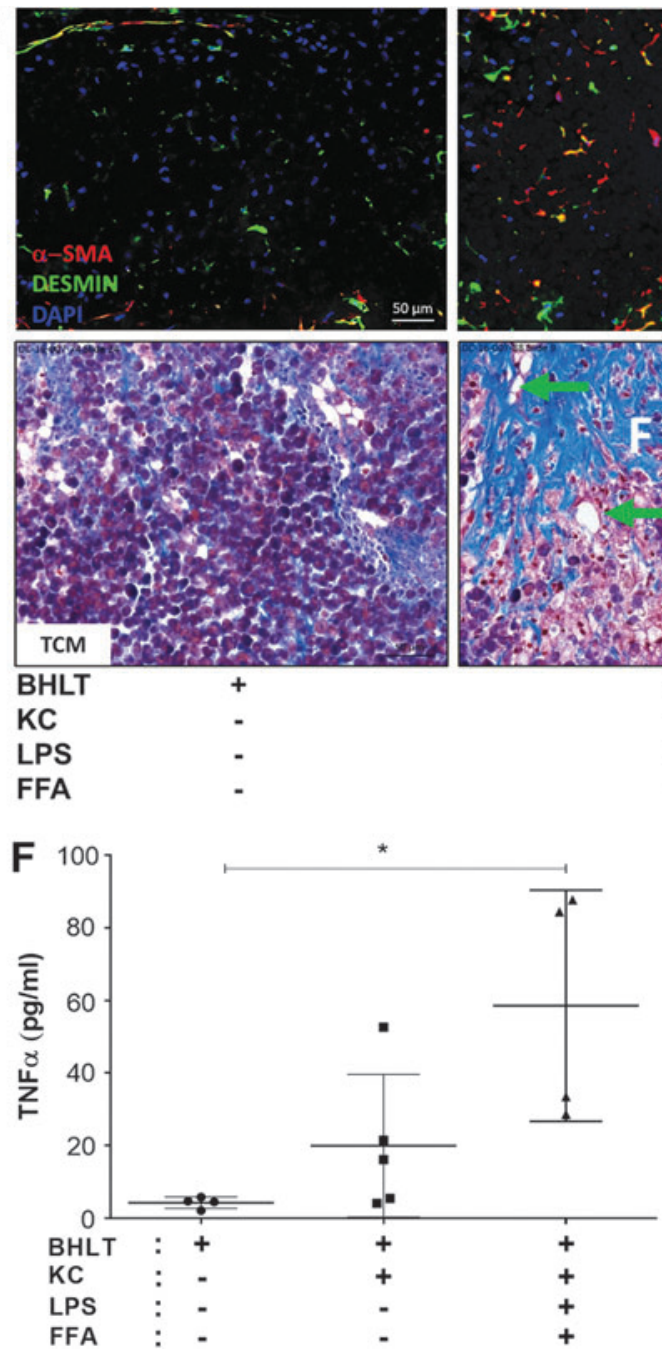
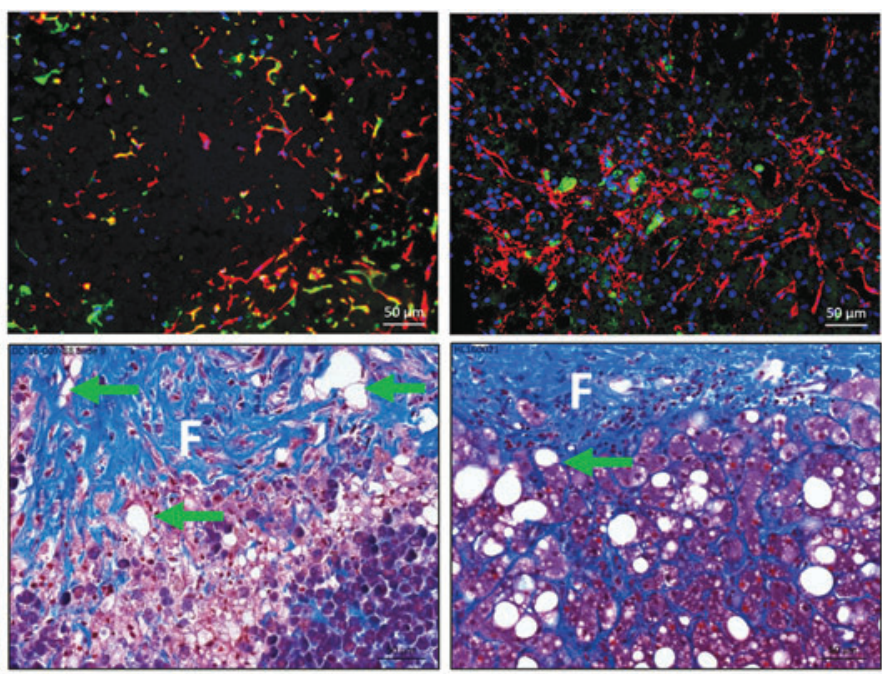

$+$

$+$

$+$

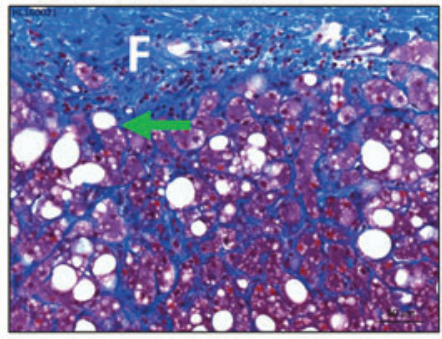

Native NASH

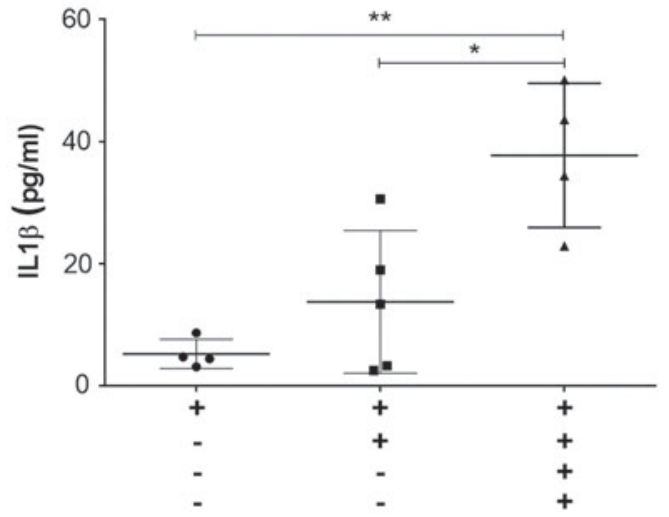

FIG. 6. (Continued).

\section{Case Study 5: Establishing Disease-Like Phenotype with Disease-Origin Cells}

The role of donor origin and associated genetic and epigenetic programming has been the focus of investigation for cells that are used in any cell-based application, including hepatocytes, mesenchymal stem cells, iPSC, and myriad other cell types. ${ }^{58-61}$ In one study, primary human lung fibroblasts were isolated from healthy donors or from idiopathic pulmonary fibrosis patients. The isolated disease-origin fibroblasts displayed a senescent phenotype, shortened telomeres, and an aberrant response to stimulation with TGF $\beta .^{62}$ The prevailing hypothesis is that isolated cells retain features of the donor and tissue of origin and will therefore be variably susceptible to stimuli, such as differentiation factors, injurious agents, or pathogens. Following this logic, cells isolated from a human liver with NAFLD/ NASH and fibrosis would potentially retain the ability to express disease-associated features after isolation and in vitro culture.

To test this hypothesis, disease-origin NPCs were substituted for standard healthy-origin cells in BHLT using the same tissue design. HSC, KC and liver endothelial cells (LEC) were obtained from human tissue donors with confirmed NAFLD/NASH and fibrosis and incorporated with standard healthy donor hepatocytes to generate tissues (C. Khatiwala and L. Hazelwood, unpublished data). The disease-origin cells formed a cohesive tissue similar to tissues constructed from healthy-origin cells and replicated key features of healthy-origin BHLT, such as the presence of small microvascular structures, formation of numerous intercellular relationships, and a compartmentalized architecture with distinct zones enriched for either hepatocytes or NPCs (Fig. 7A, B). However, the disease-origin tissues rapidly developed substantial fibrosis without the inclusion of exogenous stimuli, such as TGF $\beta$, high sugar or fat, or profibrotic drugs, and displayed phenotypic features generally aligned with the tissue of origin (Fig. 7C). Gene expression analysis of replicate diseaseorigin BHLT revealed strong expression of fibrosis-associated genes and strong concordance between samples (Fig. 7D). These data suggest that cells may carry aspects of diseased donor phenotypes into BHLT. Additional studies are required to determine which NPC types (LEC, KC, and HSC) are responsible for specific features of the disease-origin BHLT.

\section{Discussion}

As highlighted by the above case studies, BHLT can be applied in vitro across a spectrum of applications in the drug development process. As highlighted in recent reviews, 
A

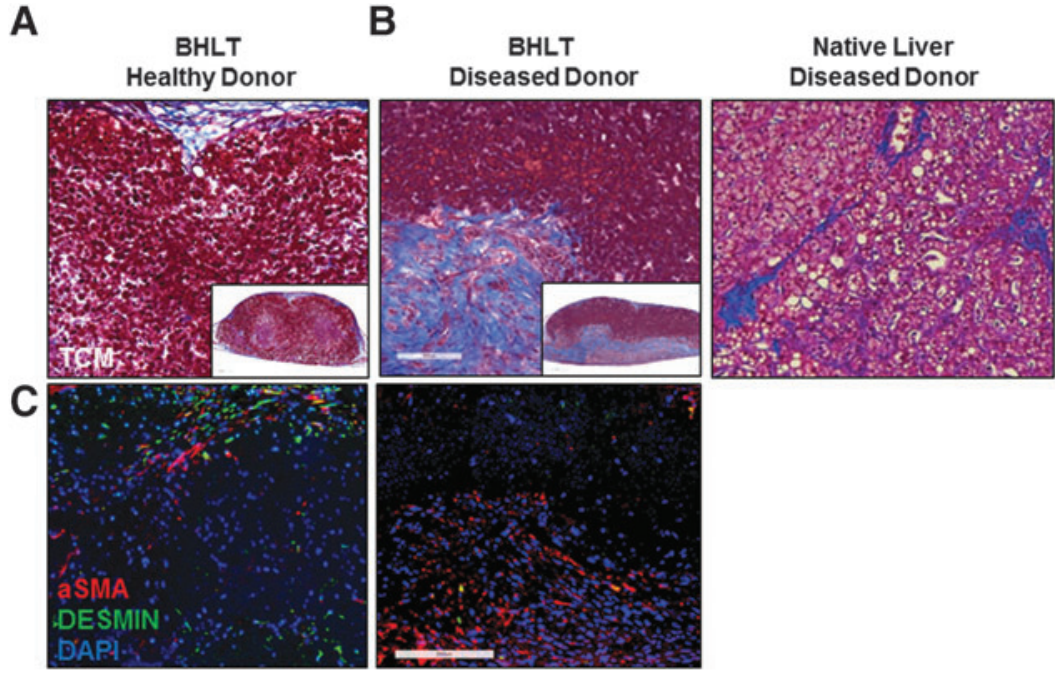

D

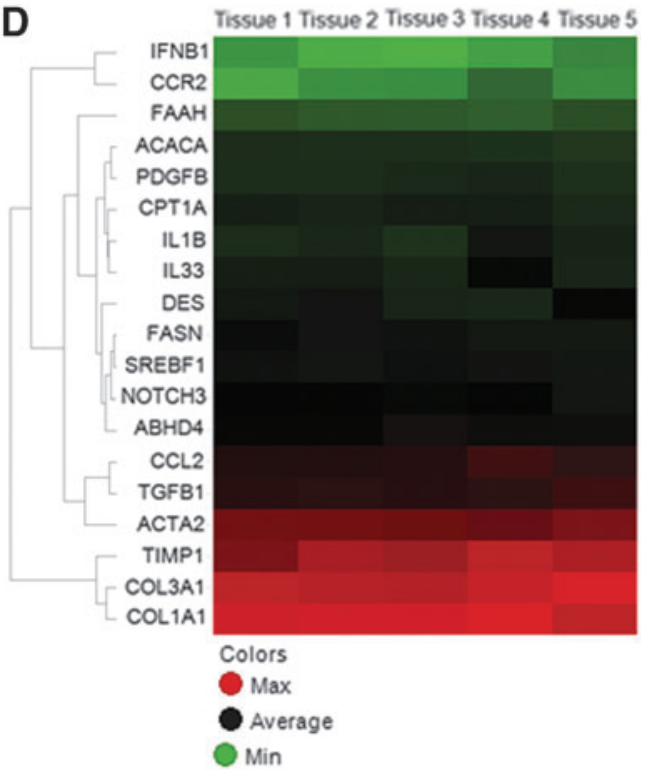

FIG. 7. Use Case Study 5: Impact of disease-origin cells on tissue phenotype. BHLT were fabricated using primary human hepatocytes from a healthy donor, and NPCs (HSC, EC, and KC) from either (A) healthy donors or (B) a donor with confirmed NASH and fibrosis. (A, B) FFPE tissues at 14 days post-fabrication were subjected to trichrome staining and (C) des$\min ^{+}$(green) and $\alpha-\mathrm{SMA}^{+}$(red) immunohistochemistry to highlight collagen deposition and stellate cell activation, respectively. (D) Quantitative RT-PCR normalized to GAPDH was performed on (five) disease-origin tissue replicates. Hierarchical clustering analysis ( $\log 10$ transformation, Spotfire software) revealed robust expression of fibrosis-associated genes and a high degree of reproducibility among samples.

each in vitro approach to modeling human liver has some advantages and limitations that must be considered when they are applied. ${ }^{63,64}$ While no one model system provides a comprehensive solution across all applications, such as toxicity screening, investigational toxicity, or efficacy on a background of disease or injury, a fit-for-purpose model is selected based on its ability to address a key-specific question. In Table 1, we summarize the performance, advantages, and limitations of BHLT across the most common applications in vitro.

BHLT are metabolically competent and able to accurately identify known hepatotoxicants in vitro, differentiate between toxic drugs and safe alternatives, and enable mechanistic investigations. The overall size of a typical BHLT enables each tissue to be used in the isolation of nucleic acids or proteins as well as histological characterization to aid in analysis. Furthermore, the longevity of the system enables the collection of longitudinal data, that is, biochemical data collected over time from the same sample such that each unique tissue can be traced temporally, analogous to serum chemistry data in preclinical animal studies. In the case of acute or even some chronic hepatocellular toxicity applications, there may be limited need for histological data as the biochemical and/ or molecular readouts may be adequate for resolution. Thus, the relatively low throughput and higher cost of BHLT and similar 3D tissue-based systems may impede their adoption over established, inexpensive high-throughput models such as cell lines or hepatocyte monocultures.

The value of BHLT in the assessment of compound-related toxicity is much greater when subacute/chronic exposure is necessary ( $\geq 2$ weeks), and the mechanism of liver injury involves complex interactions with hepatocytes and other cell types. For example, the interplay between $\mathrm{KC}$ and hepatocytes influences aspects of inflammation-mediated liver injury, and fibrotic liver injury relies on the presence and activation of HSC adjacent to hepatocytes. When a cell type is hypothesized to be responsible for mediating a toxic response, BHLT provide a platform to empirically determine the role of that cell in the response by conducting parallel experiments with and without the cell type. Exposure of BHLT to the highsugar/high FFA conditions described in Case Study 4 and Figure 6 required the inclusion of $\mathrm{KC}$ for the ultimate development of progressive steatosis and fibrosis, and thus, we can deduce that KC are likely to be key players in the development and/or progression of NAFLD and NASH secondary to dietrelated injury. Applications that involve longer term exposure and/or multicellular mechanisms are more likely to benefit from histological endpoints that reveal outcomes at the cellular level within the spatial context of the tissue architecture and its intercellular relationships. For example, the general tissue injury response to MTX that was described by Norona et al. was evident biochemically, with elevations in lactate dehydrogenase (LDH) and ALT; however, the activation and migration of stellate cells and pericellular fibrosis that developed between hepatocytes were detected through examination of the tissue by histology and required spatial context for interpretation. ${ }^{14}$ Although BHLT are not replicas of native human liver (i.e., they do not have lobular architecture or sinusoidal blood flow and lack systemic immune cells and cholangiocytes), they do establish key cell-cell relationships between $\mathrm{HC}, \mathrm{KC}, \mathrm{EC}$, and HSC and create a predictable tissue architecture that can be assessed for multiple injury-induced phenotypic changes that correlate with well-described forms of tissue damage (e.g., necrosis, tissue dissociation, cellular 
Table 1. Application-Specific Performance, Advantages, and Limitations of Bioprinted Human Liver Tissues

\begin{tabular}{|c|c|c|c|}
\hline Application & Model performance & Potential advantages & Potential limitations \\
\hline $\begin{array}{l}\text { Acute } \\
\text { hepatocellular } \\
\text { toxicity }\end{array}$ & Competent $^{12,13,28-30}$ & Biomass; Histological confirmation & Throughput; Cost \\
\hline $\begin{array}{l}\text { Chronic } \\
\text { hepatocellular } \\
\text { toxicity }\end{array}$ & Competent $^{13,14,28-30,35, *}$ & $\begin{array}{l}\text { Biomass; Longitudinal biochemical } \\
\text { data; Histological confirmation }\end{array}$ & Throughput; Cost \\
\hline $\begin{array}{l}\text { Toxicity with } \\
\text { multicellular } \\
\text { mechanism }\end{array}$ & Competent $^{12-14,28-30,35, *}$ & $\begin{array}{l}\text { Biomass; Longitudinal data; } \\
\text { Controlled mechanistic studies; } \\
\text { (+/- cell types); Histological } \\
\text { confirmation }\end{array}$ & $\begin{array}{l}\text { Throughput; Not compatible with } \\
\text { standard modes of real-time cell- } \\
\text { specific data acquisition }\end{array}$ \\
\hline $\begin{array}{l}\text { Toxicity with } \\
\text { histological } \\
\text { endpoint(s) }\end{array}$ & Competent $^{12-14,28-30,35, *}$ & $\begin{array}{l}\text { Biomass; Multiple human cell types } \\
\text { in } 3 \mathrm{D} \text { (HC+NPC); Majority of } \\
\text { tissues not in direct contact with } \\
\text { culture plastics; Compatible with } \\
\text { histological assays; Compatible } \\
\text { with ancillary biochemical/ } \\
\text { molecular assays }\end{array}$ & $\begin{array}{l}\text { Not compatible with standard } \\
\text { modes of real-time cell-specific } \\
\text { data acquisition; Need to sacrifice } \\
\text { tissues for longitudinal histological } \\
\text { analyses; BHLT are not exact } \\
\text { replicas of in vivo liver }\end{array}$ \\
\hline
\end{tabular}

Pharmacokinetic/ Limited
pharmacodynamic molecular assays

Multiple human cell types in 3D (HC+NPC); Potential to measure tissue response versus cellular response

Limited understanding of dose translation; IVIVE; Limited understanding of compound interaction with nontissue aspects of system (i.e., media components, plastics); Limited understanding of exposure levels per cell within 3D structure; Current systems lack dynamic fluid flow

Disease modeling, Strong ${ }^{13,14, *} \quad$ Biomass; Incorporation of HSC, induced fibrosis

$\mathrm{KC}$, and EC; Ability to detect activation $\leftrightarrow$ deactivation in HSC; Responds to spectrum of inducers (MTX, TAA, TGF $\beta$ ); Fibrogenesis detected by signal transduction, gene expression, immunohistochemistry, and histological stains for insoluble (fibrillar) collagens

Biomass; Incorporation of HSC, $\mathrm{KC}$, and EC; Ability to detect activation $\leftrightarrow$ deactivation in HSC; Responds to multiple high-fat/highsugar regimens $+/$ - inflammatory+; Persistent steatosis measurable biochemically and histologically; Develops all hallmarks of NAFLD/ NASH (steatosis, ballooning degeneration, inflammatory response, and fibrosis)

Disease modeling, Assessment diseased-origin in progress cells
Biomass; Disease-origin cells easily substituted into standard model; Inducer-free development of disease feature (progressive fibrosis); Works across multiple donors
Not compatible with standard modes of real-time cell-specific data acquisition; Need to sacrifice tissues for longitudinal histological analyses; BHLT are not exact replicas of in vivo liver

Not compatible with standard modes of real-time cell-speciflic data acquisition; Need to sacrifice tissues for longitudinal histological analyses; BHLT are not exact replicas of in vivo liver

Not compatible with standard modes of real-time cell-specific data acquisition; Need to sacrifice tissues for longitudinal histological analyses; BHLT are not exact replicas of in vivo liver

\footnotetext{
*Norona et al., "Bioprinted human liver provides insight into the role of Kupffer cells in modulating early events in TGFB1 and methotrexate-induced fibrotic injury," submitted.

BHLT, bioprinted human liver tissues; EC, endothelial cells; HC, hepatocytes; HSC, hepatic stellate cells; IVIVE, in vitro/in vivo extrapolation; KC, Kupffer cells; MTX, methotrexate; NAFLD, nonalcoholic fatty liver disease; NASH, nonalcoholic steatohepatitis; NPC, nonparenchymal cell; TAA, thioacetamide; TGF $\beta$, transforming growth factor beta; 3D, three-dimensional.
} 
migration, vascular remodeling, steatosis, fibrosis). Immunohistochemical or in situ hybridization analyses of tissue sections enable further assessment of cellular level responses within the context of the BHLT as a whole. Due to the overall size and complexity of BHLT and the current limitations of cell labeling and imaging technologies ${ }^{65}$ real-time tracking and functional assessment of individual cells within the tissues are not yet feasible; thus, replicate tissues must be sacrificed for histological analyses to glean cell-specific information with spatial orientation.

Perhaps one of the most challenging applications for any in vitro system is pharmacodynamic/pharmacokinetic modeling $(\mathrm{PK} / \mathrm{PD})$ and in vitro/in vivo extrapolation. To correlate compound concentrations and measured responses in vitro with doses and systemic outcomes in vivo, researchers and developers capture measurable parameters in the in vitro systems and build algorithms based on a set of assumptions to enable in vivo extrapolation (reviewed in Abaci and Shuler ${ }^{66}$ ). Algorithms are then refined and validated using a set of knowns for which both in vitro and in vivo data (animal or human) are available. Scaling from the in vitro system to in vivo assumptions is challenging and is often focused primarily on extrapolation of cell number or resident time of blood in the tissue. One may not be able to assume that every like cell within a particular model system performs identically but may exhibit heterogeneity secondary to a variety of factors, including origin, position, exposure to oxygen or nutrient gradients, and physical forces. As highlighted by Abaci and Shuler, future endeavors will likely need to include other parameters necessary for translation, such as cell density, metabolic rates, and drug partitioning. ${ }^{66}$ Like native liver, cells within BHLT are heterogeneous in phenotype and function. For example, hepatocytes closest to the culture media interface or immediately adjacent to NPC-enriched areas exhibit the greatest albumin production (similar to what is observed in periportal hepatocytes), and HSC located away from media and plastic interfaces display a more quiescent-like phenotype (unpublished data). ${ }^{14}$ Bioavailability is another confounding factor that must be considered, in particular when the cells or the compound-containing fluids within the model system come in contact with the biomaterial components of the system itself [i.e., polystyrene, poly(dimethylsiloxane), and various hydrogels]. It is not uncommon for a significant portion of compound added to the system to bind either to the polymeric or to protein components of the system through the interaction of the test compound with the biomaterial surfaces, the proteins that bind to those surfaces, and the proteins found in the media. ${ }^{67}$ Consider the example of an in vitro human liver model, which continuously secretes serum proteins (many of which bind drug and plastic) into the media, such that relative quantities of protein and bioavailable drug may vary significantly in the system at any given time. Extrapolating from in vitro systems that are relatively simple in composition (one to two cell types) and consisting of juxtaposed monolayers or tissues that are two to three cell layers thick is a significant challenge; the size (in particular, the thickness) of BHLT, the cellular complexity, and the absence of directed fluid flow paths have limited their application in PK/PD to date. Future scenarios may combine larger more complex tissues such as BHLT with the elegant engineering of today's tissue chip technologies to create more integrated solutions.

As highlighted by the case studies, a strength of BHLT is their ability to produce histological endpoints to report out a tissue-level response to injury or treatment, providing information that would not be gleaned from traditional in vitro biochemical or molecular studies. The vascular injury response to MCT was uniquely detected in the 3D environment of the BHLT along with the activation and migration of HSC and the intimate structural relationships that developed between the pericyte-like $\mathrm{SMA}^{+} \mathrm{HSC}$ and the branching, hypertrophic vascular structures. 36 Although the "systemic"like measures of tissue damage (LDH, ALT) signaled minimal liver injury, histological evaluation revealed a significant tissue response that prominently involved the NPCs. Similarly, exposure to profibrotic agents caused mild systemic signs of injury, especially at early time points, whereas histological examination revealed significant tissue injury with stellate cell activation and fibrosis that disrupted cell-cell junctions. ${ }^{14}$ The dynamic nature of BHLT is highlighted by Case Study 3 with the demonstration that the ALK5 inhibitor, galunisertib, was effective in the resolution of TGF $\beta$-induced fibrosis. ${ }^{15}$ These data suggest that BHLT are particularly useful in modeling both the transition from health to injury/disease and the transition between injured/diseased to repaired/regenerated.

The study of diet-induced liver injury (Case Study 4) is a compelling example of the use of BHLT to model a complex liver disease state (NAFLD/NASH), which today requires histopathological scoring for a definitive clinical diagnosis. $53,57,68$ In addition to producing biochemical evidence of liver damage, BHLT subjected to high-sugar and high FFA feeding regimens develop the hallmark morphological signs of NAFLD/NASH, including steatosis, ballooning degeneration of hepatocytes, inflammation (in the form of cytokine release from the comprising cells), and fibrosis. ${ }^{11,12}$ With the tissue-level phenotypic evidence supporting the existence and progression of diseaserelevant diagnostic features, it is feasible to interpret biochemical and molecular endpoints with confidence and engage in the testing of compounds targeted at the treatment of NAFLD and NASH. Moreover, with the high prevalence of NAFLD and $\mathrm{NASH}$ in the general population, it may be advantageous to test the safety of new drugs in models with a relevant disease background, such as BHLT with NASH phenotype. It is highly likely that NAFLD/NASH patients will be more susceptible to certain forms of drug injury due to compromised liver functions associated with the disease state. ${ }^{69}$ By building BHLT from cells derived from human donors with specific disease states, as highlighted in Case Study 5, it is feasible to recapitulate disease-associated phenotypes driven primarily by the genetic and epigenetic attributes of the cellular building blocks, without the requirement for exogenous inducers. Such models will be useful in the testing of candidate drugs for the disease by uniquely enabling mechanistic studies of disease progression in vitro in a human tissue system.

The majority of in vitro cell-based screens for drug safety conducted today employ traditional monocellular hepatocyte cultures to assess the cellular (hepatocyte) response to injury but do not address the contributions of other liver cell types. Where do BHLT fit within the realm of preclinical testing? If BHLT were mechanistically studied using a reductionist approach at the cellular level by separately taking each component, culturing as a 2D monocellular system, and exposing each to the same stimulus as the whole BHLT, one would likely get very different outcomes. In vitro studies with BHLT are analogous to preclinical animal studies in which serum chemistry data provide systemic hints of whether 
the drug is causing significant liver injury during the period of exposure, but it is not until the animals are biopsied or sacrificed (or the BHLT is harvested and processed) and the liver tissue is examined that the systemic signal is understood at the cellular level. Model liver systems, such as BHLT, that combine tissue-like three-dimensionality with the presence of both HC and NPC provide a holistic means of assessing the tissue response to injury. Depending on the specific application, fully human BHLT may offer translational advantages over preclinical animal models; thus, they are perhaps best viewed as an adjunct or alternative to animal studies.

\section{Author Disclosure Statement}

K.R., D.C., A.C., E.P., C.K., C.C.-G., and S.P. are all employees of Organovo, Inc., the manufacturer of ExVive ${ }^{\mathrm{TM}}$ human liver tissue. L.H. is an employee of AbbVie and the work reported herein was performed jointly by Organovo, Inc. and AbbVie. L.L.-M. is an employee of Bristol-Myers Squibb and the work reported herein was performed jointly by Organovo, Inc. and Bristol-Myers Squibb. U.H. was an employee of BristolMyers Squibb at the time this work was completed. L.N. was a graduate student at the University of North Carolina at Chapel Hill at the time this work was completed. Her graduate work was supported in part by funding from Organovo, Inc.

\section{References}

1. Jakab K, Neagu A, Mironov V, et al. Engineering biological structures of prescribed shape using self-assembling multicellular systems. Proc Natl Acad Sci U S A 2004:101; 2864-2869.

2. Smith CM, Stone AL, Parkhill RL, et al. Three-dimensional bioassembly tool for generating viable tissue-engineered constructs. Tissue Eng 2004:10;1566-1576.

3. Beysens DA, Forgacs G, Glazier JA. Cell sorting is analogous to phase ordering in fluids. Proc Natl Acad Sci U S A 2000: 97;9467-9471.

4. Jakab K, Neagu A, Mironov V, et al. Organ printing: Fiction or science. Biorheology 2004:41;371-375.

5. Gungor-Ozkerim PS, Inci I, Zhang YS, et al. Bioinks for 3D bioprinting: An overview. Biomater Sci 2018:6:915-946.

6. Placone JK, Engler AJ. Recent advances in extrusion-based 3D printing for biomedical applications. Adv Healthc Mater 2017:7:e1701161.

7. Zhang YS, Oklu R, Dokmeci MR, et al. Three-Dimensional Bioprinting Strategies for Tissue Engineering. Cold Spring Harb Perspect Med 2018:8;pii: a025718.

8. Nguyen DG, Pentoney SL, Jr. Bioprinted three dimensional human tissues for toxicology and disease modeling. Drug Discov Today Technol 2017:23;37-44.

9. Yasuda T, Obara H, Hsu HC, et al. Proposal of a novel evaluation index for the effects of shear stress and exposure time on hepatocyte damage. J Artif Organs 2015:18;236-242.

10. Retting KN, Nguyen DG. Additive manufacturing in the develoment of 3D skin tissues. In: Marques A, Reis R, Dirraco R, Cerqueira M (eds). Skin Tissue Models; pp. 377397. Boston: Academic Press; 2018.

11. Carter DE, Nguyen DG, Brenner DA, et al. The Liver Meeting (AASLD). November 11, 2017. Washington, D.C. Modeling NAFLD using 3D bioprinted human liver tissue. Hepatology 2017:66;1040A.

12. Carter DE, Presnell SC, Brenner DA, et al. The International Liver Congress (EASL). April 12, 2018. Paris, France.
Modeling NAFLD using 3D bioprinted human liver tissue. J Hepatology 2018:68;S357.

13. Nguyen DG, Funk J, Robbins JB, et al. Bioprinted 3D primary liver tissues allow assessment of organ-level response to clinical drug induced toxicity in vitro. PLoS One 2016: $11 ; \mathrm{e} 0158674$.

14. Norona LM, Nguyen DG, Gerber DA, et al. Editor's highlight: Modeling compound-induced fibrogenesis in vitro using three-dimensional bioprinted human liver tissues. Toxicol Sci 2016:154;354-367.

15. Paffenroth EH, Crogan-Grundy C, Echegaray C, et al. Utilization of a $3 \mathrm{D}$ bioprinted liver tissue model to evaluate to evaluate the antifibrotic effects of an ALK5 inhibitor in a TGFB-induced model of hepatic fibrosis. The Toxicologist: Supplement to Toxicological Sciences. Society of Toxicology 2018, Abstract 1383: 2018;162.

16. Bhise NS, Manoharan V, Massa S, et al. A liver-on-a-chip platform with bioprinted hepatic spheroids. Biofabrication 2016:8;014101.

17. Grix T, Ruppelt A, Thomas A, et al. Bioprinting perfusionenabled liver equivalents for advanced organ-on-a-chip applications. Genes (Basel) 2018:9;pii: E176.

18. Jeon H, Kang K, Park SA, et al. Generation of multilayered 3D structures of HepG2 cells using a bio-printing technique. Gut Liver 2017:11;121-128.

19. Kang K, Kim Y, Jeon H, et al. Three-dimensional bioprinting of hepatic structures with directly converted hepatocytelike cells. Tissue Eng Part A 2018:24;576-583.

20. Ma X, Qu X, Zhu W, et al. Deterministically patterned biomimetic human iPSC-derived hepatic model via rapid 3D bioprinting. Proc Natl Acad Sci U S A 2016:113;22062211.

21. Skardal A, Devarasetty M, Kang HW, et al. Bioprinting cellularized constructs using a tissue-specific hydrogel bioink. J Vis Exp 2016;10:e53606.

22. Cacabelos R. Parkinson's disease: From pathogenesis to pharmacogenomics. Int J Mol Sci 2017:18; pii: E551.

23. Mizuno Y, Shimoda S, Origasa H. Long-term treatment of Parkinson's disease with levodopa and other adjunctive drugs. J Neural Transm (Vienna) 2018:125;35-43.

24. Borges N. Tolcapone in Parkinson's disease: Liver toxicity and clinical efficacy. Expert Opin Drug Saf 2005:4; 69-73.

25. Olanow CW, Watkins PB. Tolcapone: An efficacy and safety review (2007). Clin Neuropharmacol 2007:30;287294.

26. Longo DM, Yang Y, Watkins PB, et al. Elucidating differences in the hepatotoxic potential of tolcapone and entacapone with DILIsym(®), a mechanistic model of drug-induced liver injury. CPT Pharmacometrics Syst Pharmacol 2016: $5 ; 31-39$.

27. Grunig D, Felser A, Bouitbir J, et al. The catechol-Omethyltransferase inhibitors tolcapone and entacapone uncouple and inhibit the mitochondrial respiratory chain in HepaRG cells. Toxicol In Vitro 2017:42;337-347.

28. Ware BR, McVay M, Sunada WY, et al. Exploring chronic drug effects on microengineered human liver cultures using global gene expression profiling. Toxicol Sci 2017:157; 387-398.

29. Ohbuchi M, Tetsuka K, Nagata M, et al. Mechanistic study of acetaminophen-induced liver injury using a 3D bioprinted human liver tissue model. The Toxicologist: Supplement to Toxicological Sciences. Society of Toxicology 2017, Abstract 1653: 2017;156, p. 1653. 
30. Tetsuka K, Ohbuchi M, Moriguchi H, et al. Functional evaluation of bioprinted human liver tissue as a liver injury model. The Toxicologist: Supplement to Toxicological Sciences. Society of Toxicology 2018, Abstract 1980: 2016;150.

31. Crogan-Grundy C, Smith TR, Smith RC, et al. Utilization of the ExVive(TM) human liver tissue model to assess drug-induced liver injury across a diverse set of chemical classes. The Toxicologist: Supplement to Toxicological Sciences. Society of Toxicology 2017, Abstract 1246: 2017;156.

32. Copple BL, Banes A, Ganey PE, et al. Endothelial cell injury and fibrin deposition in rat liver after monocrotaline exposure. Toxicol Sci 2002:65;309-318.

33. Copple BL, Ganey PE, Roth RA. Liver inflammation during monocrotaline hepatotoxicity. Toxicology 2003:190; 155-169.

34. Yee SB, Hanumegowda UM, Copple BL, et al. Endothelial cell injury and coagulation system activation during synergistic hepatotoxicity from monocrotaline and bacterial lipopolysaccharide coexposure. Toxicol Sci 2003:74;203-214.

35. Yee SB, Hanumegowda UM, Hotchkiss JA, et al. Role of neutrophils in the synergistic liver injury from monocrotaline and bacterial lipopolysaccharide exposure. Toxicol Sci 2003:72;43-56.

36. Hanumegowda UM, Wu Y, Smith TR, et al. Monocrotaline Toxicity in 3D-Bioprinted Human Liver Tissue. The Toxicologist: Supplement to Toxicological Sciences. Society of Toxicology 2016, Abstract 3662: 2016;150.

37. Mederacke I, Hsu CC, Troeger JS, et al. Fate tracing reveals hepatic stellate cells as dominant contributors to liver fibrosis independent of its aetiology. Nat Commun 2013:4;2823.

38. Zhang F, Hao M, Jin $\mathrm{H}$, et al. Canonical hedgehog signalling regulates hepatic stellate cell-mediated angiogenesis in liver fibrosis. Br J Pharmacol 2017:174;409-423.

39. Zhang F, Zhang Z, Chen L, et al. Curcumin attenuates angiogenesis in liver fibrosis and inhibits angiogenic properties of hepatic stellate cells. J Cell Mol Med 2014:18; 1392-1406.

40. Field RA, Stegelmeier BL, Colegate SM, et al. An in vitro comparison of the cytotoxic potential of selected dehydropyrrolizidine alkaloids and some N-oxides. Toxicon 2015: 97;36-45.

41. Tamta H, Pawar RS, Wamer WG, et al. Comparison of metabolism-mediated effects of pyrrolizidine alkaloids in a HepG2/C3A cell-S9 co-incubation system and quantification of their glutathione conjugates. Xenobiotica 2012:42; 1038-1048.

42. Renwick AB, Mistry H, Barton PT, et al. Effect of some indole derivatives on xenobiotic metabolism and xenobioticinduced toxicity in cultured rat liver slices. Food Chem Toxicol 1999:37;609-618.

43. DeLeve LD, Wang X, Kuhlenkamp JF, et al. Toxicity of azathioprine and monocrotaline in murine sinusoidal endothelial cells and hepatocytes: The role of glutathione and relevance to hepatic venoocclusive disease. Hepatology 1996: 23;589-599.

44. Friedman SL. Liver fibrosis-From bench to bedside. J Hepatol 2013:38(Suppl 1);S38-S53.

45. Chu X, Bleasby K, Evers R. Species differences in drug transporters and implications for translating preclinical findings to humans. Expert Opin Drug Metab Toxicol 2013:9; 237-252.
46. Martignoni M, Groothuis GM, de Kanter R. Species differences between mouse, rat, dog, monkey and human CYPmediated drug metabolism, inhibition and induction. Expert Opin Drug Metab Toxicol 2006:2;875-894.

47. Bjorkman DJ, Boschert M, Tolman KG, et al. The effect of long-term methotrexate therapy on hepatic fibrosis in rheumatoid arthritis. Arthritis Rheum 1993:36;16971701.

48. Maybury CM, Jabbar-Lopez ZK, Wong T, et al. Methotrexate and liver fibrosis in people with psoriasis: A systematic review of observational studies. Br J Dermatol 2014:171; 17-29.

49. Andersen ME, Krewski D. The vision of toxicity testing in the 21st century: Moving from discussion to action. Toxicol Sci 2010:117; 17-24.

50. Knudsen TB, Keller DA, Sander M, et al. FutureTox II: In vitro data and in silico models for predictive toxicology. Toxicol Sci 2015:143;256-267.

51. Prestigiacomo V, Weston A, Messner S, et al. Profibrotic compounds induce stellate cell activation, ECM-remodelling and Nrf2 activation in a human 3Dmulticellular model of liver fibrosis. PLoS One 2017: $12 ; \mathrm{e} 0179995$.

52. Leite SB, Roosens T, El Taghdouini A, et al. Novel human hepatic organoid model enables testing of drug-induced liver fibrosis in vitro. Biomaterials 2016:78;1-10.

53. Bataller R, Brenner DA. Liver fibrosis. J Clin Invest 2005: 115;209-218.

54. Davidson MD, Kukla DA, Khetani SR. Microengineered cultures containing human hepatic stellate cells and hepatocytes for drug development. Integr Biol (Camb) 2017:9; 662-677.

55. Kostrzewski T, Cornforth T, Snow SA, et al. Threedimensional perfused human in vitro model of nonalcoholic fatty liver disease. World J Gastroenterol 2017: $23 ; 204-215$.

56. Feaver RE, Cole BK, Lawson MJ, et al. Development of an in vitro human liver system for interrogating nonalcoholic steatohepatitis. JCI Insight 2016:1;e90954.

57. Benedict M, Zhang X. Non-alcoholic fatty liver disease: An expanded review. World J Hepatol 2017:9;715-732.

58. den Braver-Sewradj SP, den Braver MW, Vermeulen NP, et al. Inter-donor variability of phase I/phase II metabolism of three reference drugs in cryopreserved primary human hepatocytes in suspension and monolayer. Toxicol In Vitro 2016:33;71-79.

59. Heslop JA, Kia R, Pridgeon CS, et al. Donor-dependent and other nondefined factors have greater influence on the hepatic phenotype than the starting cell type in induced pluripotent stem cell derived hepatocyte-like cells. Stem Cells Transl Med 2017:6;1751.

60. Klontzas ME, Vernardis SI, Heliotis M, et al. Metabolomics analysis of the osteogenic differentiation of umbilical cord blood mesenchymal stem cells reveals differential sensitivity to osteogenic agents. Stem Cells Dev 2017:26; 723-733.

61. Vos JC, den Braver-Sewradj SP, den Braver MW, et al. Inter-individual variability in activity of the major drug metabolizing enzymes in liver homogenates of 20 individuals. Curr Drug Metab 2018:19;370-381.

62. Alvarez D, Cardenes N, Sellares J, et al. IPF lung fibroblasts have a senescent phenotype. Am J Physiol Lung Cell Mol Physiol 2017:313;L1164-L1173. 
63. LeCluyse EL, Witek RP, Andersen ME, et al. Organotypic liver culture models: Meeting current challenges in toxicity testing. Crit Rev Toxicol 2012:42;501-548.

64. Sistare FD, Mattes WB, LeCluyse EL. The promise of new technologies to reduce, refine, or replace animal use while reducing risks of drug induced liver injury in pharmaceutical development. ILAR J 2016:57;186-211.

65. Hoffman AF, Nolan J, Gebhard DF, et al. Society of biomolecular imaging and informatics high-content screening/ high-content analysis emerging technologies in biological models, when and why? Assay Drug Dev Technol 2018: $16 ; 1-6$.

66. Abaci HE, Shuler ML. Human-on-a-chip design strategies and principles for physiologically based pharmacokinetics/ pharmacodynamics modeling. Integr Biol (Camb) 2015:7; 383-391.

67. Gokaltun A, Yarmush ML, Asatekin A, et al. Recent advances in nonbiofouling PDMS surface modification strate- gies applicable to microfluidic technology. Technology (Singap World Sci) 2017:5;1-12.

68. Arias IM, Alter HI, Boyer JL. The Liver Biology and Pathobiology, 5th ed. Chichester, West Sussex: John Wiley \& Sons, Ltd.; 2009.

69. Massart J, Begriche K, Moreau C, et al. Role of nonalcoholic fatty liver disease as risk factor for drug-induced hepatotoxicity. J Clin Transl Res 2017:3(Suppl 1);212-232.

Address correspondence to:

Dr. Sharon Presnell

Organovo, Inc. 6275 Nancy Ridge Drive

Suite 110

San Diego, CA 92121

E-mail: spresnell@organovo.com 